\title{
Analysis of Hybrid Selection/Maximal-Ratio Diversity Combiners With Gaussian Errors
}

\author{
A. Annamalai and C. Tellambura, Member, IEEE
}

\begin{abstract}
The paper examines the impact of Gaussian distributed weighting errors (in the channel gain estimates used for coherent combination) on both the output statistics of a hybrid selection/maximal-ratio (SC/MRC) receiver and the degradation of the average symbol-error rate (ASER) performance as compared with the ideal case. New expressions are derived for the probability density function, cumulative distribution function and moment generating function (MGF) of the coherent hybrid SC/MRC combiner output signal-to-noise ratio (SNR). The MGF is then used to derive exact, closed-form, ASER expressions for binary and $M$-ary modulations in conjunction a nonideal hybrid SC/MRC receiver in a Rayleigh fading environment. Results for both selection combining (SC) and maximal-ratio combining (MRC) are obtained as limiting cases. Additionally, the effect of the weighting errors on both the outage rate of error probability and the average combined SNR is investigated. These analytical results provide insights into the tradeoff between diversity gain and combination losses, in concert with increasing orders of diversity branches in an energy-sharing communication system.
\end{abstract}

Index Terms-Binary and $M$-ary signaling, coherent combiner with weighting errors, diversity methods, hybrid diversity receivers.

\section{INTRODUCTION}

$\mathbf{T}$ HE HYBRID selection/maximal-ratio (SC/MRC) diversity scheme has received considerable attention, in recent literature, owing to its ability to alleviate the detrimental effects of deep fades on wireless channels. It has been found to achieve a good compromise between the receiver performance and the implementation complexity (fewer electronics as well as lower power consumption) [1]-[9]. In an $M / L-\mathrm{SC} / \mathrm{MRC}$ system, the receiver selects the $M$ strongest branches of $L$ diversity branches and then combines them coherently in an maximal-ratio combining (MRC) combiner to produce the decision statistic. Study of this hybrid SC/MRC receiver is important both practically and theoretically, because the hybrid system encapsulates the two well-known classical, nonhybrid diversity schemes selection combining (SC) $(M=1)$ and $\mathrm{MRC}$ $(M=L)$ as limiting cases. Moreover, the $M / L$-SC/MRC receiver outperforms an $M$-MRC receiver configuration due to the improved signal-to-noise ratio (SNR) statistics at the output

Manuscript received November 10, 2000; accepted June 18, 2001. The editor coordinating the review of this paper and approving it for publication is D. L. Goeckel.

A. Annamalai is with the Bradley Department of Electrical and Computer Engineering, Virginia Polytechic Institute and State University, Alexandria, VA 22314 USA (e-mail: annamalai@vt.edu).

C. Tellambura is with the School of Computer Science and Software Engineering, Faculty of Information Technology, Monash University, Clayton, Victoria 3168, Australia (e-mail: chintha@csse.monash.edu.au).

Publisher Item Identifier 10.1109/TWC.2002.800548. of the hybrid combiner [4]. On the other hand, its performance will be inferior to the ideal $L$-MRC receiver structure for any $M<L$, as one would expect. Performance analysis of the hybrid diversity receiver is much more complicated than that of classical diversity schemes because the hybrid case involves order statistics. Consequently, only a relatively limited number of analytical results have been published.

In all the above analyses [1]-[9], the channel gain estimates are assumed to be noiseless and, hence, perfect. In fact, very few published analytical results exist for the effect of channel estimation errors on the performance of classical diversity schemes, let alone for the hybrid SC/MRC scheme. Five notable contributions for the classical diversity schemes are [10]-[14]. In [10], Bello and Nelin first showed that frequency decorrelation of the pilot from the data signal exhibits a Gaussian distribution. Subsequently, the average bit-error-rate (ABER) expressions were derived for both coherent and noncoherent detection of binary orthogonal signals employing a nonideal MRC receiver in Rayleigh fading. In [11], investigation into the effects of weighting errors on the ABER was confined to the development of bounds on the reduction of the average output SNR for either a fixed-amplitude error or a fixed-phase error in the weighting factor. Proakis [12] derived ABER expressions for an $M$-ary phase-shift-keying (MPSK) signaling scheme in Rayleigh and Rician channels when an ad-hoc estimator, based on pilot signal or clairvoyant information from the data signal, is used to determine the channel weights. In [13], Gans modeled the channel estimation errors in MRC as being complex Gaussian and subsequently, the probability density function (PDF) and cumulative distribution function (CDF) of the combiner's output SNR were derived for a Rayleigh fading environment. More recently, [14] showed that, for arbitrary modulation formats that employ nonideal MRC, the ABER can be written in canonical form, i.e., as a weighted sum of $L$ ideal BER terms, each of which employs MRC with increasing diversity orders.

The major contributions of this paper include the following: 1) derivation of the PDF, $\mathrm{CDF}$ and moment generating function (MGF) of the hybrid $M / L$-SC/MRC combiner's output SNR, with due consideration for the Gaussian distribution of weighting errors in the channel gain estimates; 2) derivation of closed-form average symbol-error rate (ASER) formulas for a broad class of modulation formats that are used for nonideal hybrid SC/MRC; and 3) derivation of analytical expressions for computing both the outage rate of error probability for several common modulation formats and the average combiner output SNR. To the best of the authors' knowledge, study of the effect of weighting errors on the PDF, CDF, and MGF of the 


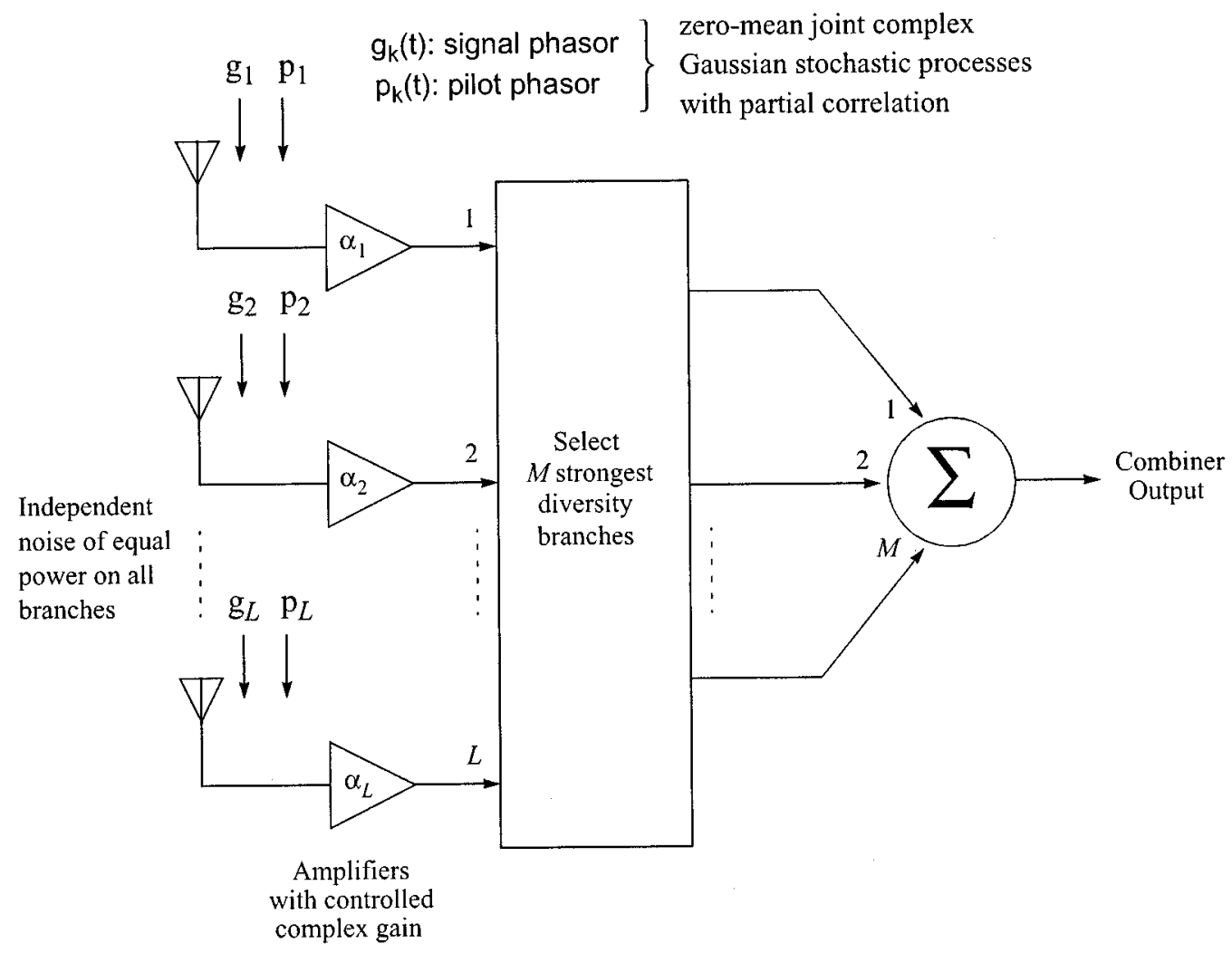

$$
\begin{array}{ll}
\text { Maximal-Ratio Combining }(M=L): & \alpha_{k}=p_{k}{ }^{*} \\
\text { Selection Combining }(M=1): & \alpha_{k}= \begin{cases}1 & \text { if }\left|p_{k}\right| \geq\left|p_{i}\right| \text { for all } i \\
0 & \text { otherwise }\end{cases}
\end{array}
$$

Fig. 1. Hybrid $M / L-\mathrm{SC} / \mathrm{MRC}$ predetection combiner.

hybrid SC/MRC combiner output SNR is not available in related literature. Investigation into the effect of Gaussian errors on the SC receiver also appears to be new. Furthermore, neither closed-form expressions for the ASER of arbitrary modulations formats derived for the SC/MRC receiver, nor their corresponding outage rates have been reported previously. Finally, it is not entirely clear if the complexity of combiner and the combination losses (due to weighting errors in the gain estimates) will outweigh the benefits obtained from additional diversity branches in an energy sharing communication system. Insights into this are also given in this paper.

This organization of this paper as follows. In Section II, we obtain the PDF, CDF, and MGF of the SC/MRC combiner output SNR for independent and identically distributed (i.i.d.) diversity branches, in a Rayleigh fading environment and Gaussian distributed weighting errors in the channel gain estimates. Closed-form expressions for the ASER of a wide range of modulation formats are derived in Section III using the MGF. In Section IV, an analysis of the outage rate of error probability, which utilizes the CDF, is presented for several common modulation formats. An expression for the statistical average of the combiner output SNR is also derived using the MGF. Several important limiting cases are investigated in Section V. Where applicable, we show that our expressions reduce to the well-known results in the literature. Selected numerical results are provided in Section VI and this is followed by a summary of the major results in Section VII.

\section{Derivation of Hybrid SC/MRC COMbINER OUtPut STATISTICS}

In this section, we will derive the PDF, CDF, and MGF of a nonideal $M / L$-SC/MRC combiner output SNR for i.i.d. Rayleigh fading channels. This is done by assuming that the weighting error is of the complex Gaussian type. The hybrid combiner is illustrated schematically in Fig. 1. The complex gain $\alpha_{k}$ of each branch in the combiner is controlled either by estimating the signal on each branch or by detecting a pilot that was sent along with the signal. For this reason, the Gaussian errors may be due either to inaccurate signal estimation (e.g., obtained via a clairvoyant estimator, as used in [12]) or due to decorrelation between the pilot and the signal arising from too large a frequency or time separation [13]. For instance, in the case of the MRC combiner, $\alpha_{k}=p_{k}^{*}$ where the pilot signal of the $k$ th branch $p_{k}$ differs from the data signal $g_{k}$ by a random error, whose real and imaginary parts have a Gaussian distribution with a zero-mean, and where the asterisk indicates complex conjugate. This is equivalent to assuming 
that the real and imaginary parts of $g_{k}$ and $p_{k}$ are jointly Gaussian distributed with partial correlation, as when the pilot tone has a frequency separation from the signal carrier by an amount greater than or equal to the coherence bandwidth of the channel. Readers may refer to [13] (see also [26]) for a detailed explanation of the system model under consideration. As far as possible, we will also attempt to follow the notation used in [13].

Similar to the treatment in [13], we have defined several real random variables in terms of the complex random variables $p_{k}$ and $g_{k}$, in order to divide the SNR into parts that are either dependent or independent of the noise (which is determined by the $\left.r_{k}\right)$

$$
\begin{aligned}
p_{k}= & r_{k} \exp \left(j \theta_{k}\right) \\
g_{k}= & {\left[\left(x_{k}+\frac{R_{c} r_{k}}{\sigma_{x}^{2}}\right)+j\left(y_{k}+\frac{R_{c s} r_{k}}{\sigma_{x}^{2}}\right)\right] } \\
& \times \exp \left(j \theta_{k}\right) \equiv r_{g k} \exp \left[j\left(\theta_{k}+\varphi_{k}\right)\right]
\end{aligned}
$$

which defines $r_{k}, \theta_{k}, x_{k}, y_{k}, r_{g k}$, and $\varphi_{k}$ as real quantities, and

$$
\begin{aligned}
& \sigma_{x}^{2}=\left\langle\left(\Re p_{k}\right)^{2}\right\rangle=\left\langle\left(\Re g_{k}\right)^{2}\right\rangle=\left\langle\left(\Im g_{k}\right)^{2}\right\rangle=\left\langle\left(\Im p_{k}\right)^{2}\right\rangle \\
& R_{c}=\left\langle\left(\Re p_{k}\right)\left(\Re g_{k}\right)\right\rangle=\left\langle\left(\Im p_{k}\right)\left(\Im g_{k}\right)\right\rangle \\
& R_{c s}=\left\langle\left(\Re p_{k}\right)\left(\Im g_{k}\right)\right\rangle=-\left\langle\left(\Re g_{k}\right)\left(\Im p_{k}\right)\right\rangle \\
& \left\langle\left(\Re p_{k}\right)\left(\Im p_{k}\right)\right\rangle=\left\langle\left(\Re g_{k}\right)\left(\Im g_{k}\right)\right\rangle=0
\end{aligned}
$$

where notation $\langle\cdot\rangle$ denotes the ensemble average, $x_{k}$ and $y_{k}$ are joint Gaussian distributed with zero-mean and a variance of $\sigma_{x}^{2}-\left(R_{c}^{2}+R_{c s}^{2}\right) / \sigma_{x}^{2}, \theta_{k}$ is the locally generated carrier phase estimate of the data signal while $\varphi_{k}$ is the carrier phase error (i.e., the difference in the carrier phase between the data signal and pilot) of the $k$ th branch. In the above definitions, we have assumed equal statistics for each independent diversity branches, and the correlation between pilot and signal are the same on each branch. Since the gain on all branches may be changed in common without affecting the SNR, there is no loss of generality by setting the variance of the pilot phasor equal to that of the signal phasor. Moreover, Gans [13] has elegantly showed that the definitions (1) and (2) ensure that the random variables $x_{k}$ and $y_{k}$ are uncorrelated with $r_{k}$. This property greatly simplifies the derivation of the PDF of combiner output SNR, as we will show next.

From the above definitions, it can be readily shown that the output SNR $\gamma$ is given by [13, eq. (19)]

$$
\gamma=\frac{(X+x)^{2}+(Y+y)^{2}}{2 N}
$$

where $N$ represents the average noise power on each branch

$$
\begin{array}{r}
x=\frac{\sum_{k=1}^{M} r_{(k)} x_{k}}{\sqrt{\sum_{k=1}^{M} r_{(k)}^{2}}, \quad y=\frac{\sum_{k=1}^{M} r_{(k)} y_{k}}{\sqrt{\sum_{k=1}^{M} r_{(k)}^{2}}},} \\
X=\frac{R_{c}}{\sigma_{x}^{2}} \sqrt{\sum_{k=1}^{M} r_{(k)}^{2}}, \quad Y=\frac{R_{c s}}{R_{c}} X
\end{array}
$$

and $r_{(k)}$ denotes the $k$ th order statistic (i.e., obtained after rearranging the random variables $r_{1}, \ldots, r_{L}$ in a descending order such that $\left.r_{(1)} \geq r_{(2)} \geq \cdots \geq r_{(L)}\right)$. If the pilot and the data signal are not highly correlated, then the likelihood of not always choosing the $M$ "strongest" diversity branches (i.e., highest SNR) increases, which translates into degradation of the $M / L-\mathrm{SC} / \mathrm{MRC}$ performance in comparison to the ideal case. This performance loss may be quantified by deriving the PDF and CDF of the random variable $\gamma$ described in (3). For any given set of $\left\{r_{(k)}\right\}$, it is not difficult to demonstrate that $x$ and $y$ are joint Gaussian distributed and independent of which set of $\left\{r_{(k)}\right\}$ we choose. Consequently, we can compute the PDF of combiner output SNR by convolving the density distributions of its independent parts or by exploiting a property of the noncentral chi-square statistics.

To facilitate the derivation of the PDF of the combiner output SNR, we define a random variable

$$
Z=X^{2}+Y^{2}=\frac{R_{c}^{2}+R_{c S}^{2}}{\sigma_{x}^{4}} \sum_{k=1}^{M} r_{(k)}^{2}
$$

and then perform an averaging of the conditional probability $p_{\gamma}(\gamma \mid Z)$ taken over the PDF of $Z$, viz.

$$
p_{\gamma}(\gamma)=\int_{-\infty}^{\infty} p_{\gamma}(\gamma \mid Z) p_{Z}(Z) d Z
$$

where $p_{\gamma}(\gamma \mid Z)$ can be manipulated from [13, eq. (91)] to yield

$p_{\gamma}(\gamma \mid Z)=\frac{N}{\sigma_{0}^{2}} \exp \left(-\frac{2 N \gamma+Z}{2 \sigma_{0}^{2}}\right) I_{0}\left(\frac{\sqrt{2 N \gamma Z}}{\sigma_{0}^{2}}\right), \quad \gamma \geq 0$

and the PDF of $Z$ is given by (see Appendix I)

$$
\begin{aligned}
& p_{Z}(Z)= \\
& \begin{cases}\sum_{l=0}^{L-M} \sum_{k=1}^{D_{l}} \mu_{l}^{\left(D_{l}-k\right)} \frac{Z^{k-1}}{(k-1) ! \lambda_{l}^{k}} \exp \left(-Z / \lambda_{l}\right), & Z \geq 0 \\
0, & Z \leq 0\end{cases}
\end{aligned}
$$

where $\sigma_{0}^{2}=\left(\sigma_{x}^{4}-R_{c}^{2}-R_{c s}^{2}\right) / \sigma_{x}^{2}, I_{0}($.$) is the modified Bessel$ function of the first kind of order zero

$$
D_{l}= \begin{cases}M, & \text { for } l=0 \\ 1, & \text { for } l \neq 0\end{cases}
$$

$\mu_{l}^{\left(D_{l}-k\right)}$ corresponds to the coefficients of partial fractions, $\lambda_{l}=$ $2 \sigma^{2}(M /(l+M))$ and $\sigma^{2}=\left(R_{c}^{2}+R_{c s}^{2}\right) / \sigma_{x}^{2}$. It is also worth mentioning that (5) can be derived directly from (3) by recognizing that the random variable $2 N \gamma$ conditioned on $Z$ has a noncentral chi-square distribution with noncentrality parameter $X^{2}+Y^{2}=Z$ because $x$ and $y$ are i.i.d. Gaussian random variables with zero-mean and a variance $\sigma_{0}^{2}$.

Substituting (5) and (6) into (4), with some additional simplifications, we obtain (see Appendix II)

$$
\begin{aligned}
p_{\gamma}(\gamma)= & \sum_{l=0}^{L-M} \sum_{k=1}^{D_{l}} \frac{\mu_{l}^{\left(D_{l}-k\right)}}{\Lambda_{l}^{k}}\left[\bar{\gamma}\left(1-\rho^{2}\right)\right]^{k-1} \sum_{n=0}^{k-1} \frac{1}{n !} \\
& \times\left(\begin{array}{c}
k-1 \\
n
\end{array}\right)\left[\frac{M \rho^{2} \gamma}{\Lambda_{l}\left(1-\rho^{2}\right)(l+M)}\right]^{n} \exp \left(-\frac{\gamma}{\Lambda_{l}}\right)
\end{aligned}
$$


where $\Lambda_{l}=\bar{\gamma}\left[\left(M+l\left(1-\rho^{2}\right)\right) /(l+M)\right], \rho$ denotes the correlation between the actual complex channel gains and their estimates, $\bar{\gamma}$ is the average SNR/symbol per branch, and the coefficients $\mu_{l}^{(0)}$ and $\mu_{0}^{(M-k)}$ may be computed as

$$
\begin{aligned}
& \mu_{l}^{(0)}=\prod_{i=0, i \neq l}^{L-M}\left(\frac{i+M}{i-l}\right)^{D_{i}} \\
& = \begin{cases}\left(\begin{array}{c}
L \\
M
\end{array}\right), & \text { for } l=0 \\
(-1)^{M+l-1}\left(\frac{M}{l+M}\right) & \\
\left(\frac{M}{l}\right)^{M-1}\left(\begin{array}{c}
L \\
M
\end{array}\right)\left(\begin{array}{c}
L-M \\
l
\end{array}\right), & \text { for } l \neq 0\end{cases} \\
& \mu_{0}^{(M-k)}= \begin{cases}\sum_{i=l}^{L-M}(-1)^{i+M-k-1}\left(\begin{array}{c}
L \\
M
\end{array}\right) & \\
\left(\begin{array}{c}
L-M \\
i
\end{array}\right)\left(\frac{M}{i}\right)^{M-k}, & \text { if } k<M<L \\
0, & \text { if } k<M \\
\text { and } M=L .\end{cases}
\end{aligned}
$$

Following this, the CDF of the hybrid SC/MRC combiner output SNR, which has Gaussian distributed weighting errors, is given by

$$
\begin{aligned}
F_{\gamma}(\gamma)= & -\sum_{l=0}^{L-M} \sum_{k=1}^{D_{l}} \exp \left(-\frac{\gamma}{\Lambda_{l}}\right)\left[\frac{\bar{\gamma}\left(1-\rho^{2}\right)}{\Lambda_{l}}\right]^{k-1} \mu^{\left(D_{l}-k\right)} \\
& \times \sum_{n=0}^{k-1}\left(\begin{array}{c}
k-1 \\
n
\end{array}\right)\left[\frac{M \rho^{2}}{\left(1-\rho^{2}\right)(l+M)}\right]^{n} \sum_{w=0}^{n} \frac{1}{w !}\left(\frac{\gamma}{\Lambda_{l}}\right)^{w}
\end{aligned}
$$

using [19, eqs. (8.350.1) and (8.352.1)]. Taking Laplace transform of the PDF (7), we get the MGF of the combiner output SNR (with the aid of identity [19, eq. (3.351.3)])

$$
\begin{aligned}
\phi_{\gamma}(s) & =\sum_{l=0}^{L-M} \sum_{k=1}^{D_{l}} \mu_{l}^{\left(D_{l}-k\right)}\left[\frac{\bar{\gamma}\left(1-\rho^{2}\right)}{\Lambda_{l}}\right]^{k-1} \sum_{n=0}^{k-1} \\
& \times\left(\begin{array}{c}
k-1 \\
n
\end{array}\right)\left[\frac{M \rho^{2}}{\left(1-\rho^{2}\right)(l+M)}\right]^{n} \frac{1}{\left(1+s \Lambda_{l}\right)^{n+1}} .
\end{aligned}
$$

We believe that the above results [(7), (10), and (11)] are an improvement on the existing results for the hybrid SC/MRC output SNR statistics since the existing results have been generalized by considering the impact of Gaussian distributed weighting errors on a variety of receiver structures. It is also important to note that [24] independently derived the MGF of SC/MRC output SNR with channel decorrelation using a slightly different approach. Application of these new results in the characterization of the performance of diversity systems in terms of ASER, outage rate of error probability and the average combined SNR is presented in Sections III and IV.

\section{ASER}

In this section, we will show that it is possible to derive ASER expressions (in closed-form) for arbitrary, two-dimensional $M$-ary signals that have polygonal decision regions, $M$-ary DPSK and $\pi / 4$-DQPSK modulation schemes, among others. We consider the nonideal SC/MRC receiver on Rayleigh fading channels. The key to our derivation is a combination of factors, $v i z$, application of the MGF technique (originally described in [20] for binary phase-shift keying (BPSK), $M$-ary PSK, and $M$-ary DPSK schemes that have diversity reception) and the availability of solutions to the three generic trigonometric integrals furnished in [15]. Concisely, the final results will be summarized below. Nevertheless, it should be pointed out that these expressions can be obtained simply by inspecting the modulation-dependent parameters in the conditional error probability (i.e., the error probability in an AWGN channel) that is expressed in a desirable exponential form. Alternatively, one may inspect the arguments of the MGF and the integration limits. Readers may refer to [15] and [23] for further details on this approach. By applying the MGF technique, we are able to demonstrate that for a broad class of binary and $M$-ary modulations that employ an SC/MRC receiver with Gaussian weighting errors, the ASER can be expressed in terms of the MGF alone, given by (11). For instance, the ASER performance of an arbitrary two-dimensional signal constellations [21], [22] may be computed as

$$
\begin{aligned}
\overline{P_{e}}= & \frac{1}{2 \pi} \sum_{z=1}^{S} W_{z} \int_{0}^{\eta_{z}} \phi_{\gamma}\left[\frac{a_{z} \sin ^{2}\left(\varphi_{z}\right)}{\sin ^{2}\left(\theta+\varphi_{z}\right)}\right] d \theta \\
= & \sum_{l=0}^{L-M} \sum_{k=1}^{D_{l}} \mu_{l}^{\left(D_{l}-k\right)}\left[\frac{\bar{\gamma}\left(1-\rho^{2}\right)}{\Lambda_{l}}\right]^{k-1} \\
& \times \sum_{n=0}^{k-1}\left(\begin{array}{c}
k-1 \\
n
\end{array}\right)\left[\frac{M \rho^{2}}{\left(1-\rho^{2}\right)(l+M)}\right]^{n} \\
& \times \frac{1}{2 \pi} \sum_{z=1}^{S} W_{z} \int_{0}^{\eta_{z}}\left[1+\frac{\Lambda_{l} a_{z} \sin ^{2}\left(\varphi_{z}\right)}{\sin ^{2}\left(\theta+\varphi_{z}\right)}\right]^{-(n+1)} d \theta
\end{aligned}
$$

where $S$ is the total number of signal points or decision subregions, $W_{z}$ is the a priori probability of the symbol to which subregion $z$ corresponds, $a_{z}, \eta_{z}$, and $\varphi_{z}$ are coefficients (constants) relating to decision subregion $z$.

Recognizing that the integral in (12) has a known closed-form solution [15], the above expression can be computed conveniently using (13)

$$
\begin{aligned}
\overline{P_{e}}= & \sum_{l=0}^{L-M} \sum_{k=1}^{D_{l}} \mu_{l}^{\left(D_{l}-k\right)}\left[\frac{\bar{\gamma}\left(1-\rho^{2}\right)}{\Lambda_{l}}\right]^{k-1} \\
& \times \sum_{n=0}^{k-1}\left(\begin{array}{c}
k-1 \\
n
\end{array}\right)\left[\frac{M \rho^{2}}{\left(1-\rho^{2}\right)(l+M)}\right]^{n} \\
& \times \frac{1}{2 \pi} \sum_{z=1}^{S} W_{z} I_{S}\left[\varphi_{z}, \eta_{z}+\varphi_{z}, \Lambda_{l} a_{z} \sin ^{2} \varphi_{z}, n+1\right]
\end{aligned}
$$


where

$$
\begin{aligned}
I_{S}\left(\theta_{L}, \theta_{U}, a, b\right) \\
=\int_{\theta_{L}}^{\theta_{U}}\left[\frac{\sin ^{2} \theta}{a+\sin ^{2} \theta}\right]^{b} d \theta=\left(\theta_{U}-\theta_{L}\right) \\
\quad+\sum_{n=1}^{b}(-1)^{n} \sum_{r=0}^{n-1} \frac{a^{n-r-1 / 2}}{(1+a)^{n-1 / 2}}\left(\begin{array}{c}
b \\
n
\end{array}\right)\left(\begin{array}{c}
n-1 \\
r
\end{array}\right) \\
\quad \times P\left(\sqrt{\frac{1+a}{a}} \tan \theta_{L}, \sqrt{\frac{1+a}{a}} \tan \theta_{U}, 1+r\right), \\
\quad \text { for integer } b \geq 1
\end{aligned}
$$

and

$$
\begin{aligned}
& P(a, b, n) \\
& =\frac{(2 n-3) ! !}{2^{n-1}(n-1) !}\left(\tan ^{-1} b-\tan ^{-1} a\right)+\frac{(2 n-3) ! !}{(n-1) !} \sum_{k=1}^{n-1} \\
& \quad \times \frac{(n-k-1) !}{2^{k}(2 n-2 k-1) ! !}\left[\frac{b}{\left(1+b^{2}\right)^{n-k}}-\frac{a}{\left(1+a^{2}\right)^{n-k}}\right] \\
& \quad n \geq 1
\end{aligned}
$$

where $(2 n-3) ! !=1.3 \cdots(2 n-3)$ and $(n-1) !=1.2 .3 \cdots(n-$ 1).

Similarly, closed-form ASER expressions for coherent BPSK, coherent binary frequency-shift keying (CFSK), noncoherent binary FSK (NCFSK), binary differential PSK (DPSK), quadrature PSK (QPSK), $M_{c}$-ary square quadrature amplitude modulation $\left(M_{c}\right.$-QAM), star-QAM, $M_{c}$-ary PSK $\left(M_{c}\right.$-PSK), $M_{c}$-ary DPSK $\left(M_{c}\right.$-DPSK), noncoherent $M_{c}$-ary FSK $\left(M_{c}\right.$-FSK) and DQPSK with Gray coding in conjunction with an $M / L-\mathrm{SC} / \mathrm{MRC}$ receiver, can all be derived in a similar fashion. The results derived, thus, are summarized below.

\section{A. NCFSK and DPSK}

$$
\begin{aligned}
\overline{P_{e}}=\frac{1}{2} \phi_{\gamma}(a)= & \sum_{l=0}^{L-M} \sum_{k=1}^{D_{l}} \frac{\mu_{l}^{\left(D_{l}-k\right)}\left(\bar{\gamma} / \Lambda_{l}\right)^{k-1}}{2\left(1+a \Lambda_{l}\right)} \\
& \times\left[1-\rho^{2}+\frac{M \rho^{2}}{(l+M)\left(1+a \Lambda_{l}\right)}\right]^{k-1}
\end{aligned}
$$

where $a=1$ for DPSK and $a=1 / 2$ for NCFSK.

\section{B. BPSK, CFSK, QPSK, and $M_{c}-P S K$}

$$
\begin{aligned}
\overline{P_{e}}= & \frac{1}{\pi} \sum_{l=0}^{L-M} \sum_{k=1}^{D_{l}} \mu_{l}^{\left(D_{l}-k\right)}\left[\frac{\bar{\gamma}\left(1-\rho^{2}\right)}{\Lambda_{l}}\right]^{k-1} \\
& \times \sum_{n=0}^{k-1}\left(\begin{array}{c}
k-1 \\
n
\end{array}\right)\left[\frac{M \rho^{2}}{\left(1-\rho^{2}\right)(l+M)}\right]^{n} \\
& \times I_{S}\left[0, \theta_{H}, \zeta \Lambda_{l}, n+1\right]
\end{aligned}
$$

where $I_{S}(\cdot, \cdot, \cdot, \cdot)$ is defined in (14), the coefficients $\theta_{H}$ and $\zeta$ for these four different modulation formats are listed in Table I, and $M_{c}$ denotes the size of $M$-ary signal constellations.
TABLE I

MOdUlation PARAMETERS FOR SEVERAL COHERENT DETECTION SCHEMES

\begin{tabular}{c|c|c}
\hline Modulation & $\theta_{H}$ & $\zeta$ \\
\hline BPSK & $\pi / 2$ & 1 \\
\hline BFSK & $\pi / 2$ & $1 / 2$ \\
\hline QPSK & $3 \pi / 4$ & $1 / 2$ \\
\hline$M_{c}$-PSK & $\pi-\pi / M_{c}$ & $\sin ^{2}\left(\pi / M_{c}\right)$ \\
\hline
\end{tabular}

C. Noncoherent $M_{c}-F S K$

$$
\overline{P_{e}}=\sum_{\nu=1}^{M_{c}-1} \frac{(-1)^{\nu+1}}{\nu+1}\left(\begin{array}{c}
M_{c}-1 \\
\nu
\end{array}\right) \phi_{\gamma}\left(\frac{\nu}{\nu+1}\right)
$$

where $\phi_{\gamma}(\cdot)$ is given in (11). It is also apparent that (18) reduces to (16) when $M_{c}=2$.

D. $M_{c}-Q A M$ and $Q P S K$

$$
\begin{aligned}
\overline{P_{e}}= & \sum_{l=0}^{L-M} \sum_{k=1}^{D_{l}} \mu_{l}^{\left(D_{l}-k\right)}\left[\frac{\bar{\gamma}\left(1-\rho^{2}\right)}{\Lambda_{l}}\right]^{k-1} \\
& \times \sum_{n=0}^{k-1}\left(\begin{array}{c}
k-1 \\
n
\end{array}\right)\left[\frac{M \rho^{2}}{\left(1-\rho^{2}\right)(l+M)}\right]^{n} \\
& \times \frac{4 q}{\pi}\left\{I_{S}\left[0, \pi / 2, \frac{3 \Lambda_{l}}{2\left(M_{c}-1\right)}, n+1\right]\right. \\
& \left.\quad q I_{S}\left[0, \pi / 4, \frac{3 \Lambda_{l}}{2\left(M_{c}-1\right)}, n+1\right]\right\}
\end{aligned}
$$

where $q=1-1 / \sqrt{M_{c}}$. Noting that signal constellations for 4-QAM and QPSK are virtually identical, the average SER for QPSK may also be obtained by substituting $M_{c}=4$ in (19). However, a more concise solution is illustrated in (17).

\section{E. $M_{c}$-DPSK, Noncoherent Detection of Equiprobable,} Correlated Binary Signals and $\pi / 4-D Q P S K$

$$
\begin{aligned}
\overline{P_{e}}= & \sum_{l=0}^{L-M} \sum_{k=1}^{D_{l}} \mu_{l}^{\left(D_{l}-k\right)}\left[\frac{\bar{\gamma}\left(1-\rho^{2}\right)}{\Lambda_{l}}\right]^{k-1} \\
& \times \sum_{n=0}^{k-1}\left(\begin{array}{c}
k-1 \\
n
\end{array}\right)\left[\frac{M \rho^{2}}{\left(1-\rho^{2}\right)(l+M)}\right]^{n} \frac{\varepsilon}{\pi} \\
& \times I_{V}\left[0, \theta_{H}, \Omega, \zeta \Lambda_{l}, n+1\right]
\end{aligned}
$$

where

$$
\begin{aligned}
I_{V}\left(\theta_{L}, \theta_{U}, c, a, b\right) \\
=\int_{\theta_{L}}^{\theta_{U}}\left(\frac{1+c \cos \theta}{1+a+c \cos \theta}\right)^{b} d \theta \\
=\left(\theta_{U}-\theta_{L}\right)+2 \sum_{n=1}^{b}\left(\begin{array}{c}
b \\
n
\end{array}\right)\left(\frac{a}{c-a-1}\right)^{n} \\
\quad \times \sum_{r=0}^{n-1} \sqrt{\frac{1+a-c}{1+a+c}}\left(\begin{array}{c}
n-1 \\
r
\end{array}\right)\left(\frac{-2 c}{1+a+c}\right)^{r} \\
\quad \times P\left[\sqrt{\frac{1+a-c}{1+a+c}} \tan \frac{\theta_{L}}{2}, \sqrt{\frac{1+a-c}{1+a+c}} \tan \frac{\theta_{U}}{2}, 1+r\right],
\end{aligned}
$$$$
\text { for integer } b \geq 1
$$ 
TABLE II

MOdULATION PARAMETERS FOR SEVERAL INCOHERENT DETECTION SCHEMES

\begin{tabular}{l|c|c|c|c}
\hline Modulation & $\varepsilon$ & $\theta_{H}$ & $\Omega$ & $\zeta$ \\
\hline$M_{c}$-DPSK & 1 & $\pi-\pi / M_{c}$ & $\cos \left(\pi / M_{c}\right)$ & $\sin ^{2}\left(\pi / M_{c}\right)$ \\
\hline $\begin{array}{l}\text { Correlated Binary Signals } \\
(\vartheta: \text { magnitude of cross }\end{array}$ & $1 / 2$ & $\pi$ & $-\vartheta$ & $\frac{1-\vartheta^{2}}{2}$ \\
correlation between the two signals $)$ & & & & \\
\hline$\pi / 4$-DQPSK & $1 / 2$ & $\pi$ & $-1 / \sqrt{2}$ & 1 \\
\hline
\end{tabular}

and coefficients $\varepsilon, \theta_{H}, \Omega$ and $\zeta$ for these modulation formats are tabulated in Table II.

We conclude this section by noting that our developments rely on the fact that the MGF (11) has a similar form to those in [15] and the fact that closed-form solutions are available for the generic trigonometric integrals $I_{S}(\cdot, \cdot, \cdot, \cdot)$ and $I_{V}(\cdot, \cdot, \cdot, \cdot, \cdot)$ derived in [15].

\section{Outage Rate of Error Probability and the Average COMBINED SNR}

Outage rate of error probability (hereafter, simply referred as outage probability) is another useful performance measure of diversity systems. The outage probability $P_{\text {out }}$ is defined as the probability that the instantaneous symbol error probability of the system will exceed a specified value (say $P_{e}^{*}$ ). This is equivalent to calculating the probability that the combiner output SNR will fall below a predetermined threshold $\gamma^{*}$ (a coefficient that is dependent on the modulation type), viz.

$$
P_{\text {out }}=\int_{0}^{\gamma^{*}} p_{\gamma}(\gamma) d \gamma=F_{\gamma}\left(\gamma^{*}\right)
$$

which is essentially the CDF of output SNR evaluated at $\gamma^{*}$. As such, the outage probability can be evaluated efficiently using (10). The threshold $\gamma^{*}$ can be obtained by solving $P_{e}\left(\gamma^{*}\right)=P_{e}^{*}$, where $P_{e}($.$) corresponds to the SER in a nonfading channel. A$ closed-form solution for $\gamma^{*}$ is available for several modulation schemes and these forms are listed below

Case a) If $P_{e}(\gamma)=a \exp (-b \gamma)$, then $\gamma^{*}=\ln \left(a / P_{e}^{*}\right) / b$

Case b) If $P_{e}(\gamma)=\operatorname{aerfc}(\sqrt{b \gamma})$, then $\gamma^{*}=(1 / b)$ $\left[\operatorname{erfc}^{-1}\left(P_{e}^{*} / a\right)\right]^{2}$

Case c) If $P_{e}(\gamma)=\operatorname{aerfc}(\sqrt{b \gamma})-\operatorname{cerfc}^{2} \sqrt{b \gamma}$, then

$$
\gamma^{*}=\frac{1}{b}\left\{\operatorname{erfc}^{-1}\left[\frac{a-\sqrt{a^{2}-4 c P_{e}^{*}}}{2 c}\right]\right\}^{2}
$$

where notation $\operatorname{erfc}^{-1}($.$) denotes the inverse of the$ complementary error function. For completeness, we summarize the common modulation schemes that take the above forms and their corresponding threshold $\gamma^{*}$ in Table III.

Next, we will derive a simple expression for the mean combined SNR at the output of an imperfect SC/MRC receiver by utilizing either the PDF or the MGF of the SNR. Since the mean
TABLE III

THRESHOLD SNR/SYMBOL $\gamma^{*}$ IN CLOSED-FORM FOR Several Common Modulation Schemes

\begin{tabular}{l|l|l}
\hline $\begin{array}{l}\text { Modulation/ } \\
\text { Detection }\end{array}$ & $\begin{array}{l}\text { Conditional Error } \\
\text { Probability } P_{e}(\gamma)\end{array}$ & $\begin{array}{l}\text { Threshold SNR/ } \\
\text { symbol } \gamma^{*}\end{array}$ \\
\hline BPSK & $\frac{1}{2} \operatorname{erfc}(\sqrt{\gamma})$ & {$\left[\operatorname{erfc}^{-1}\left(2 P_{e}^{*}\right)\right]^{2}$} \\
\hline CFSK & $\frac{1}{2} \operatorname{erfc}(\sqrt{\gamma / 2})$ & $2\left[\operatorname{erfc}^{-1}\left(2 P_{e}^{*}\right)\right]^{2}$ \\
\hline NCFSK & $\frac{1}{2} \exp (-\gamma / 2)$ & $-2 \ln \left(2 P_{e}^{*}\right)$ \\
\hline DPSK & $\frac{1}{2} \exp (-\gamma)$ & $-\ln \left(2 P_{e}^{*}\right)$ \\
\hline QPSK & $\operatorname{erfc}(\sqrt{\gamma / 2})-\frac{1}{4} \operatorname{erfc}^{2}(\sqrt{\gamma / 2})$ & $2\left\{\operatorname{erfc}^{-1}\left[2\left(1-\sqrt{1-P_{e}^{*}}\right)\right]\right\}^{2}$ \\
\hline$M_{c}$-QAM & $2 \operatorname{eerfc}\left(\sqrt{\frac{3 \gamma}{2\left(M_{c}-1\right)}}\right)$ & $\frac{2\left(M_{c}-1\right)}{3}\left(\operatorname{erfc}^{-1}\left(\frac{1-\sqrt{1-P_{e}^{*}}}{1-1 / \sqrt{M_{c}}}\right)\right)^{2}$ \\
& $-q^{2} \operatorname{erfc}^{2}\left(\sqrt{\frac{3 \gamma}{2\left(M_{c}-1\right)}}\right)$ & \\
\hline MhK & $\operatorname{erfc}(\sqrt{\gamma / 2})-\frac{1}{4} \operatorname{erfc}^{2}(\sqrt{\gamma / 2})$ & $2\left\{\operatorname{erfc}^{-1}\left[2\left(1-\sqrt{1-P_{e}^{*}}\right)\right]\right\}^{2}$ \\
\hline coherent detection of & $\operatorname{erfc}(\sqrt{\gamma})-\frac{1}{2} \operatorname{erfc}^{2}(\sqrt{\gamma})$ & {$\left[\operatorname{erfc}^{-1}\left(1-\sqrt{1-P_{e}^{*}}\right)\right]^{2}$} \\
differentially encoded & & \\
BPSK & & \\
\hline
\end{tabular}

combined SNR $\bar{\gamma}_{g s c}$ is the first moment (mean) of the random variable $\gamma$, we obtain

$$
\begin{aligned}
\bar{\gamma}_{g s c}= & \int_{0}^{\infty} \gamma p_{\gamma}(\gamma) d \gamma \\
= & -\left.\frac{d}{d s} \phi_{\gamma}(s)\right|_{s=0} \\
= & \sum_{l=0}^{L-M} \sum_{k=1}^{D_{l}} \frac{\mu_{l}^{\left(D_{l}-k\right)}}{\Lambda_{l}^{k-2}}\left[\bar{\gamma}\left(1-\rho^{2}\right)\right]^{k-1} \sum_{n=0}^{k-1}(n+1) \\
& \times\left(\begin{array}{c}
k-1 \\
n
\end{array}\right)\left[\frac{M \rho^{2}}{\left(1-\rho^{2}\right)(l+M)}\right]^{n}
\end{aligned}
$$

For the special case of $\rho=1$ (i.e., ideal SC/MRC), the above expression numerically agrees with those obtained in [2] and $[8]$.

\section{SeVERAl Important Limiting CASES}

In this section, we will examine several limiting cases, and demonstrate, where applicable, that our expressions collapses into the well-known expressions found in existing literature. The four important limiting cases of interest are as follows: 1) $\rho=1$; 2) $\rho=0$; 3) $\mathrm{MRC}(M=L)$; and 4) $\mathrm{SC}(M=1)$. Specifically, the results for the SC limiting case, which uses imperfect combining, appears to be new. Before proceeding further, we would like to point out that the MGF (11) can be rewritten more concisely as ${ }^{1}$

$$
\begin{aligned}
\phi_{\gamma}(s)= & \sum_{l=0}^{L-M} \sum_{k=1}^{D_{l}} \frac{\mu_{l}^{\left(D_{l}-k\right)}}{\left(1+s \Lambda_{l}\right)}\left[\frac{\bar{\gamma}}{\Lambda_{l}}\left(1-\rho^{2}\right)+\frac{\xi_{l} \rho^{2}}{\Lambda_{l}\left(1+s \Lambda_{l}\right)}\right]^{k-1} \\
= & \sum_{k=1}^{M} \frac{\mu_{0}^{(M-k)}}{(1+s \bar{\gamma})^{k}}\left[\left(1-\rho^{2}\right) s \bar{\gamma}+1\right]^{k-1} \\
& +\sum_{l=1}^{L-M} \frac{\mu_{l}^{(0)}}{1+s \bar{\gamma}\left[\frac{M+l\left(1-\rho^{2}\right)}{l+M}\right]}
\end{aligned}
$$

\footnotetext{
${ }^{1}$ The last summation term in (11) can be grouped together using identity $(x+$ $y)^{n}=\sum_{k=0}^{n}\left(\begin{array}{c}n \\ k\end{array}\right) x^{n-k} y^{k}$.
} 
where $\xi_{l}=\bar{\gamma} M /(l+M)$ and $\Lambda_{l}=\bar{\gamma}\left[\left(M+l\left(1-\rho^{2}\right)\right) /(l+M)\right]$. The above forms allow us to examine the limiting cases more conveniently.

\section{A. Case $\rho=1$}

Letting $\rho=1$ (coherent combining with perfect channel gain estimates) in (24), we get

$$
\begin{aligned}
\phi_{\gamma}(s) & =\sum_{l=0}^{L-M} \sum_{k=1}^{D_{l}} \mu_{l}^{\left(D_{l}-k\right)}\left(\frac{1}{1+s \xi_{l}}\right)^{k} \\
& =\prod_{l=0}^{L-M}\left(\frac{1}{1+s \xi_{l}}\right)^{D_{l}}
\end{aligned}
$$

by noting that $\mu_{l}^{\left(D_{l}-k\right)}$ is the coefficient of the partial fraction expansion, i.e.,

$$
\begin{aligned}
& \mu_{l}^{\left(D_{l}-k\right)}=\frac{\xi_{l}^{k-D_{l}}}{\left(D_{l}-k\right) !} \frac{\partial^{\left(D_{l}-k\right)}}{\partial t} \\
& \times\left.\left[\prod_{i=0, i \neq l}^{L-M}\left(\frac{1}{1+\xi_{i} t}\right)^{D_{i}}\right]\right|_{t=-1 / \xi_{l}} .
\end{aligned}
$$

Equation (25) agrees with that of [9, eq. (12)] after using a variable substitution to change the limits of the product term

$$
\begin{aligned}
\phi_{\gamma}(s) & =\frac{1}{(1+s \bar{\gamma})^{M-1}} \prod_{l=0}^{L-M} \frac{1}{1+\frac{s \bar{\gamma} M}{1+M}} \\
& =(1+s \bar{\gamma})^{1-M} \prod_{l=M}^{L} \frac{1}{1+\frac{s \bar{\gamma} M}{l}} .
\end{aligned}
$$

a) MRC

Further substituting $M=L$ in (25), we obtain a familiar expression for the MGF of the SNR at the MRC combiner output with $L$ i.i.d. diversity branches in a Rayleigh fading environment because $\mu_{0}^{(0)}=1$ and $\mu_{0}^{(L-k)}=0$ for all $k=1,2, \ldots, L-1$.

b) $\mathrm{SC}$

Now, letting $M=1$ in (25), we get the well-known expression -for MGF of SNR at the SC combiner output:

$$
\begin{aligned}
\phi_{\gamma}(s) & =\sum_{l=0}^{L-1} \mu_{l}^{(0)}\left(\frac{l+1}{l+1+s \bar{\gamma}}\right) \\
& =\sum_{l=0}^{L-1}(-1)^{l}\left(\begin{array}{c}
L \\
l+1
\end{array}\right)\left(\frac{l+1}{l+1+s \bar{\gamma}}\right)
\end{aligned}
$$

since $\mu_{0}^{(0)}=L$ and $\mu_{l}^{(0)}=(-1)^{l}(L /(l+1))\left(\begin{array}{c}L-1 \\ l\end{array}\right)$ for $l=1,2, \ldots, L-1$.

Similarly, substituting $\rho=1$ in (7) and (10), we get

$$
\begin{aligned}
p_{\gamma}(\gamma)= & \sum_{l=0}^{L-M} \sum_{k=1}^{D_{l}} \frac{\mu_{l}^{\left(D_{l}-k\right)} \gamma^{k-1}}{(k-1) ! \xi_{l}^{k}} \exp \left(-\frac{\gamma}{\xi_{l}}\right) \\
= & \sum_{k=1}^{M} \frac{\mu_{0}^{(M-k)} \gamma^{k-1}}{(k-1) ! \bar{\gamma}^{k}} \exp \left(-\frac{\gamma}{\bar{\gamma}}\right) \\
& +\sum_{l=1}^{L-M} \frac{\mu_{l}^{(0)}(l+M)}{\bar{\gamma} M} \exp \left(-\frac{\gamma(l+M)}{\bar{\gamma} M}\right)
\end{aligned}
$$

$$
\begin{aligned}
F_{\gamma}(\gamma)= & -\sum_{l=0}^{L-M} \sum_{k=1}^{D_{l}} \exp \left[-\frac{\gamma(l+M)}{\bar{\gamma} M}\right] \\
& \times \mu_{l}^{\left(D_{l}-k\right)} \sum_{w=0}^{k-1} \frac{1}{w !}\left[\frac{\gamma(l+M)}{\bar{\gamma} M}\right]^{w} \\
= & -\sum_{k=1}^{M} \mu_{l}^{(M-k)} \exp \left(-\frac{\gamma}{\bar{\gamma}}\right) \sum_{w=0}^{k-1} \frac{1}{w !}\left(\frac{\gamma}{\bar{\gamma}}\right)^{w} \\
& -\sum_{l=1}^{L-M} \mu_{l}^{(0)} \exp \left[-\frac{\gamma(l+M)}{\bar{\gamma} M}\right]
\end{aligned}
$$

because all the terms in the last sum are equal to zero except when $n=k-1$. These formulas are equivalent to those found in $[9$, eq. (16)] and [9, eq. (24)].

Letting $\rho=1$ in (23), with some additional simplifications, we obtain

$$
\begin{aligned}
\bar{\gamma}_{g s c} & =\sum_{l=0}^{L-M} \sum_{k=1}^{D_{l}} \mu_{l}^{\left(D_{l}-k\right)} \frac{k \bar{\gamma} M}{l+M} \\
& =\sum_{k=1}^{M} \mu_{0}^{(M-k)} k \bar{\gamma}+\sum_{l=1}^{L-M} \mu_{l}^{(0)} \frac{\bar{\gamma} M}{l+M}
\end{aligned}
$$

which agrees with an equivalent expression found in [8].

\section{B. Case $\rho=0$}

In the other extreme case, in which the gain estimates are completely uncorrelated with the actual branch gains, the MGF of the SC/MRC combiner output SNR (24) reduces to

$$
\phi_{\gamma}(s)=\sum_{l=0}^{L-M} \sum_{k=1}^{D_{l}} \frac{\mu_{l}^{\left(D_{l}-k\right)}}{(1+s \bar{\gamma})}=\frac{1}{(1+s \bar{\gamma})}
$$

since $\sum_{l=0}^{L-M} \sum_{k=1}^{D_{l}} \mu_{l}^{\left(D_{l}-k\right)}=1$. It is apparent that there will not be any diversity advantage for this particular case because the diversity receiver performance will be dictated by diversity branches chosen and combined randomly. Therefore, the ASER performance will be equivalent to a single channel reception (independent of both $L$ and $M$ ), which is physically intuitive. From this observation, we may conclude that there must exist an optimal value for the diversity order of energy limited communications that will minimize the ASER (for a specified, average SNR per symbol and correlation coefficient). This is because it is possible that the combination losses will outweigh the benefits obtained by having additional diversity branches (i.e., in this scenario, the average SNR/symbol per branch needs to be reduced with a higher order of diversity in order to maintain a fixed energy).

Demonstrating that (7) and (10), respectively, simplify into the PDF and CDF of the SNR of single channel reception for $\rho=0$ is very straight forward

$$
\begin{aligned}
p_{\gamma}(\gamma) & =\sum_{l=0}^{L-M} \sum_{k=1}^{D_{l}} \mu_{l}^{\left(D_{l}-k\right)} \frac{1}{\bar{\gamma}} \exp \left(-\frac{\gamma}{\bar{\gamma}}\right) \\
& =\frac{1}{\bar{\gamma}} \exp \left(-\frac{\gamma}{\bar{\gamma}}\right)
\end{aligned}
$$




$$
\begin{aligned}
F_{\gamma}(\gamma) & =1-\sum_{l=0}^{L-M} \sum_{k=1}^{D_{l}} \mu_{l}^{\left(D_{l}-k\right)} \exp \left(-\frac{\gamma}{\bar{\gamma}}\right) \\
& =1-\exp \left(-\frac{\gamma}{\bar{\gamma}}\right) .
\end{aligned}
$$

Finally, the average combined SNR (23) collapses to $\bar{\gamma}_{g s c}=$ $\bar{\gamma}$ as expected.

\section{Case $M=L$}

The MGF of the SNR at the output of an MRC combiner that has weighting errors can be readily obtained by replacing $M=L$ in (11)

$$
\begin{aligned}
\phi_{\gamma}(s)= & \sum_{k=1}^{L} \mu_{0}^{(L-k)}\left(1-\rho^{2}\right)^{k-1} \sum_{n=0}^{k-1}\left(\begin{array}{c}
k-1 \\
n
\end{array}\right) \\
& \times\left[\frac{\rho^{2}}{\left(1-\rho^{2}\right)}\right]^{n} \frac{1}{(1+s \bar{\gamma})^{n+1}} \\
= & \left(1-\rho^{2}\right)^{L-1} \sum_{t=1}^{L}\left(\begin{array}{c}
k-1 \\
t-1
\end{array}\right)\left(\frac{\rho^{2}}{1-\rho^{2}}\right)^{t-1} \frac{1}{(1+s \bar{\gamma})^{t}} \\
= & \sum_{t=1}^{L} \frac{B(t)}{(1+s \bar{\gamma})^{t}}
\end{aligned}
$$

where the weighting coefficients are Bernstein polynomials

$$
B(t)=\left(\begin{array}{c}
L-1 \\
t-1
\end{array}\right)\left(1-\rho^{2}\right)^{L-t} \rho^{2(t-1)} .
$$

The simplifications in (35) are carried out by first noting that $\mu_{0}^{(L-k)}=0$, except when $k=L$, and then using variable substitution $t=n+1$. It is evident from the last expression in (35) that when the channel weighting is not perfect, the MGF of the SNR at the output of an $L$ th order MRC combiner will simply be the weighted sum of $L$ ideal, MRC output SNR MGFs that have an increasing order. The weighting coefficients will be the Bernstein polynomials. This form, for the PDF of the combiner output SNR, was originally presented in [14]. Interestingly, the MGF (35) may also be expressed in a very compact form taken directly from (24)

$$
\phi_{\gamma}(s)=\frac{\left[\left(1-\rho^{2}\right) s \bar{\gamma}+1\right]^{L-1}}{(1+s \bar{\gamma})^{L}} .
$$

Taking the inverse Laplace transform of (35) yields the PDF [14, eq. (7a)] as expected. Alternatively, substituting $M=L$ in (7), we get

$$
\begin{aligned}
& p_{\gamma}(\gamma)=\frac{\left(1-\rho^{2}\right)^{L-1}}{\bar{\gamma}} \exp \left(-\frac{\gamma}{\bar{\gamma}}\right) \\
& \times \sum_{n=0}^{L-1} \frac{1}{n !}\left(\begin{array}{c}
L-1 \\
n
\end{array}\right)\left[\frac{\rho^{2} \gamma}{\bar{\gamma}\left(1-\rho^{2}\right)}\right]^{n}
\end{aligned}
$$

which is identical to [13, eq. (46)]. The corresponding CDF may be simplified from (10) as

$$
\begin{aligned}
F_{\gamma}(\gamma)=1-\exp & \left(-\frac{\gamma}{\bar{\gamma}}\right) \sum_{n=0}^{L-1}\left(\begin{array}{c}
L-1 \\
n
\end{array}\right) \\
& \times \rho^{2 n}\left(1-\rho^{2}\right)^{L-1-n} \sum_{w=0}^{n} \frac{1}{w !}\left(\frac{\gamma}{\bar{\gamma}}\right)^{w}
\end{aligned}
$$

and this formula agrees with [13, eq. (48)] as expected. Finally, the average combined SNR for this specific case is given by

$$
\begin{aligned}
\bar{\gamma}_{g s c} & =\bar{\gamma} \sum_{n=0}^{L-1}(n+1)\left(\begin{array}{c}
L-1 \\
n
\end{array}\right) \rho^{2 n}\left(1-\rho^{2}\right)^{L-1-n} \\
& =\bar{\gamma}\left[1+(M-1) \rho^{2}\right] .
\end{aligned}
$$

The above expression is in agreement with [13, pp. 497].

\section{Case $M=1$}

Next, we shall examine the way in which the SC receiver performance is impacted by Gaussian distributed weighting errors in the channel gain estimates. Substituting $M=1$ in (24), we obtain

$$
\begin{aligned}
\phi_{\gamma}(s) & =\frac{L}{(1+s \bar{\gamma})}+\sum_{l=1}^{L-1} \frac{(-1)^{l}\left(\begin{array}{c}
L \\
l+1
\end{array}\right)}{1+s \bar{\gamma}\left[1-l \rho^{2} /(l+1)\right]} \\
& =\sum_{l=0}^{L-1} \frac{(-1)^{l}\left(\begin{array}{c}
L \\
l+1
\end{array}\right)}{1+s \bar{\gamma}\left[1-l \rho^{2} /(l+1)\right]} .
\end{aligned}
$$

Notice that, when we either set $L=1$ or $\rho=0$, (40) reduces to the MGF of the SNR for the single channel reception case, as one would expect. As a check, we also find that we get (28) by replacing $\rho=1$ in (40). Similarly, by letting $M=1$ in (7), (10) and (23), we can, respectively, obtain simple expressions for the PDF, CDF, and average combined SNR of the SC with channel estimation errors

$$
\begin{aligned}
p_{\gamma}(\gamma) & =\sum_{l=0}^{L-1} \frac{(-1)^{l}\left(\begin{array}{c}
L \\
l+1
\end{array}\right)}{\bar{\gamma}\left[1-l \rho^{2} /(l+1)\right]} \exp \left[\frac{-\gamma}{\bar{\gamma}\left[1-l \rho^{2} /(l+1)\right]}\right] \\
F_{\gamma}(\gamma) & =1-\sum_{l=0}^{L-1}(-1)^{l}\left(\begin{array}{c}
L \\
l+1
\end{array}\right) \exp \left[\frac{-\gamma}{\bar{\gamma}\left[1-l \rho^{2} /(l+1)\right]}\right] \\
\bar{\gamma}_{g s c} & =\bar{\gamma} \sum_{l=0}^{L-1}(-1)^{l}\left(\begin{array}{c}
L \\
l+1
\end{array}\right)\left[\frac{1+l\left(1-\rho^{2}\right)}{l+1}\right]
\end{aligned}
$$

\section{COMPuTATIONAL Results AND Discussions}

In this section, we present selected numerical curves that characterize the statistics of a nonideal hybrid SC/MRC combiner output SNR and help to assess the degradation in the receiver performance due to Gaussian weighting error.

The normalized PDF of an $M / 5$-SC/MRC combiner output SNR is plotted in Fig. 2 for different $M$ and $\rho$ values. It is apparent that the probability at small $x$ values declines rapidly as $M$ increases and the mean of the distribution shifts to the right. This translates into reduced likelihood of experiencing deep fades in the wireless channels. The improvement is much more pronounced when $M$ is increased from one to two than when $M$ is increased from two to three. It is also evident that the combination losses due to imperfect branch weight estimates can affect the receiver performance considerably. For the limiting case of $\rho=0$, we find that the PDF of the output SNR is identical to that of a single channel reception because the normalized PDF curve declines exponentially with increasing $x$. 

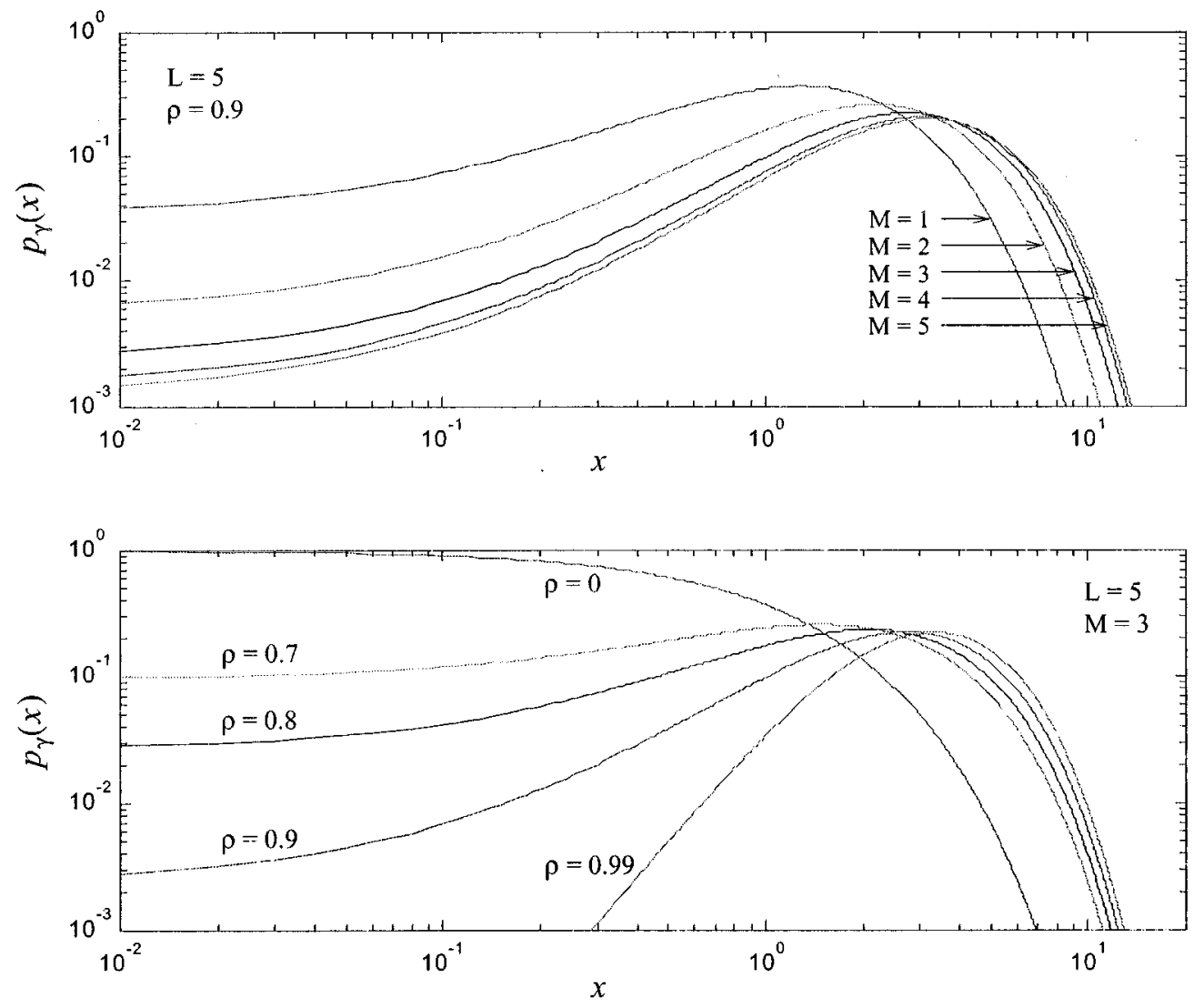

Fig. 2. Normalized PDF of $M / 5$-SC/MRC combiner output SNR.

Also, in the high $\rho$ region $(\rho \rightarrow 1)$, even a small amount of perturbation in $\rho$ will radically alter the PDF curve.

Fig. 3 depicts the CDF curves for the statistic at the output of a nonideal SC/MRC receiver. As $x \rightarrow \infty$, we observe that $F_{\gamma}(x) \rightarrow 1$, as expected. Since the outage rate error probability dictates the likelihood of instantaneous SER exceeds a specified SER, the normalized CDF curves can be used to predict the outage probability performance of diversity systems for a multitude of modulation schemes or alternatively compute the average SNR per branch to satisfy an outage requirement. For instance, the threshold SNR for DPSK is $\gamma^{*}=-\ln \left(2 \times 10^{-3}\right)=$ 6.21 (see entry 4 in Table III) given that $P_{e}^{*}=10^{-3}$. When the average SNR per branch is given, then $P_{\text {out }}$ can be readily computed as $F_{\gamma}(6.21)$ using (10). On the other hand, if $P_{\text {out }}=0.01$ is specified, then the average SNR per branch requirement may be estimated as $\bar{\gamma}=6.21 / 0.73=8.5=9.3 \mathrm{~dB}$ assuming that $L=5, M=3$ and $\rho=0.95$ [note that $x=0.73$ may be interpolated from Fig. 3 when $\left.F_{\gamma}(x)=0.01\right]$. Alternatively, by combining the four strongest diversity branches $(M=4)$ while other parameters remain unchanged, the average SNR per branch requirement reduces to $\bar{\gamma}=6.21 / 0.85=8.6 \mathrm{~dB}$ in order to satisfy the same outage criterion. The above approach may also be used for predicting the outage probability and/or the average SNR/symbol per branch for higher order alphabets. Given that $P_{e}^{*}=10^{-3}, P_{\text {out }}=0.01, L=5$ and $\rho=0.95$, it can readily computed that the average SNR/symbol per branch for QPSK is $\bar{\gamma}=10.83 / 0.73=11.7 \mathrm{~dB}$ and $\bar{\gamma}=10.83 / 0.85=$ $11 \mathrm{~dB}$ for $M=3$ and $M=4$ respectively, because $\gamma^{*}=10.83$ (using entry 5 in Table III). While higher order alphabets allow higher data transmission rates, the increased bandwidth efficiency is attained at the expense of increasing the average SNR per branch requirement (to compensate for the denser signaling constellation).

Fig. 4(a) and (b) illustrate the ASER performance of $\pi / 4$-DQPSK with Gray coding and 8-ary DPSK modulation schemes as a function of average SNR/symbol per branch for the nonideal 2/6-SC/MRC and 5/6-SC/MRC receiver configurations. While $\rho=0$, both the curves (for $M=2$ and $M=5$ ) coincide and their ASER performance is equivalent to the no diversity case, regardless of the value of $M$. This trend is in agreement with the discussion in Section V-B. Also, if $\rho<1$, the rate of decay for large average $\mathrm{SNR} / \mathrm{symbol} / \mathrm{branch}$ is identical to the single channel reception case. This observation can be explained by rewriting (24) as

$$
\begin{aligned}
\phi_{\gamma}(s)=\sum_{k=1}^{M} \mu_{0}^{(M-k)} & \frac{\left[1-\rho^{2}+1 / s \bar{\gamma}\right]^{k-1}}{s \bar{\gamma}(1+1 / s \bar{\gamma})^{k}} \\
& +\frac{1}{s \bar{\gamma}} \sum_{l=1}^{L-M} \frac{\mu_{l}^{(0)}(l+M)}{(l+M) / s \bar{\gamma}+M+l\left(1-\rho^{2}\right)}
\end{aligned}
$$

and then letting $\bar{\gamma} \rightarrow \infty$ (asymptotic analysis). It is evident that the asymptotic rate of decay is proportional to $1 / \bar{\gamma}$, as is the case with single channel reception (no diversity). In [14], the authors pointed out that the position of the knee in the ASER curve for the nonideal MRC receiver does not depend on the order of diversity $L$ but is influenced by the modulation format. We found that these trends also hold for the nonideal $M / L$-SC/MRC receiver structures. In addition, the position 

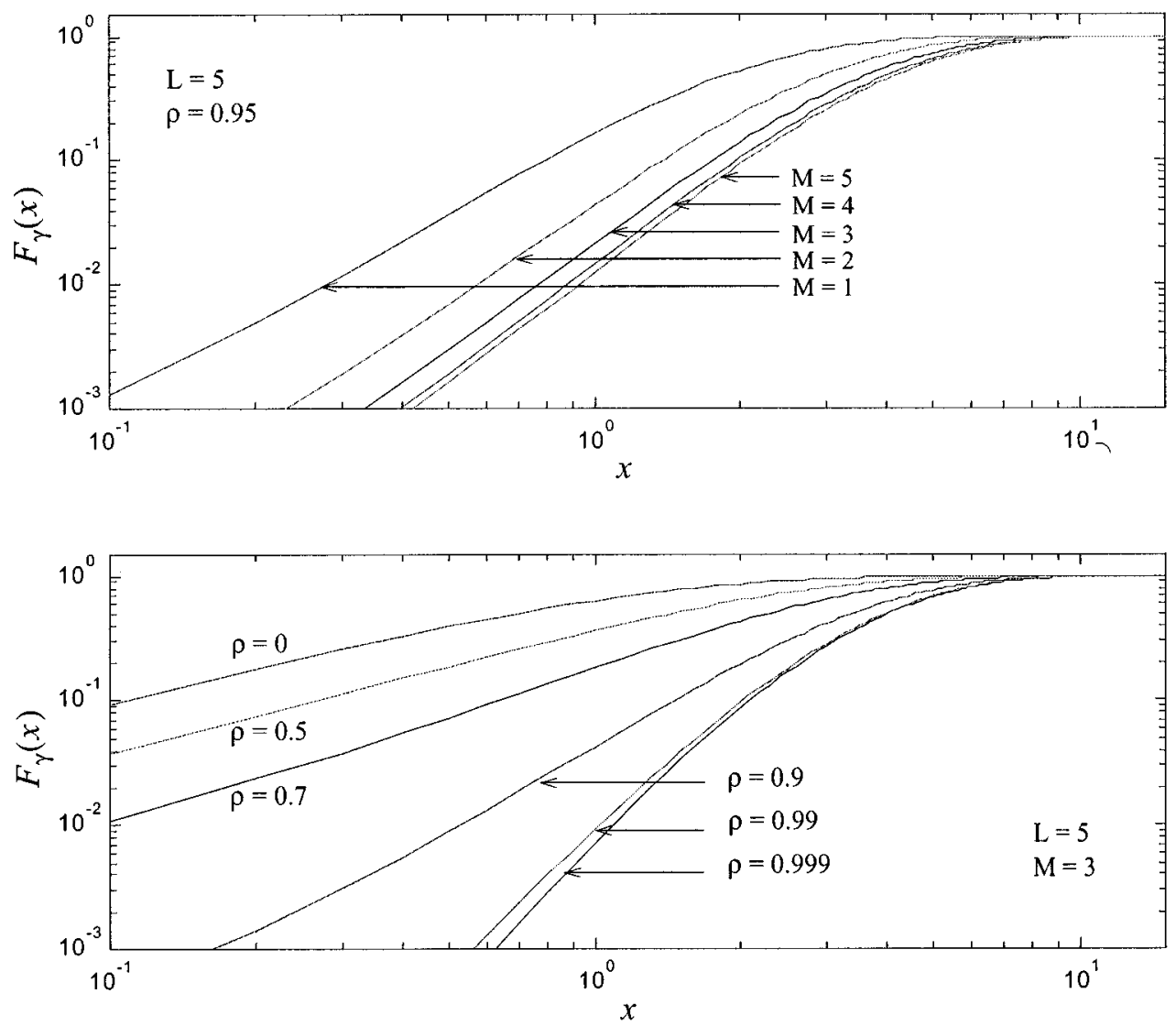

Fig. 3. Normalized CDF of $M / 5-\mathrm{SC} / \mathrm{MRC}$ combiner output SNR.

of the knee also appears not sensitive to the choice of $M$ (see also Fig. 6). Comparison between Fig. 4(a) and (b) also reveals that there is some penalty in the average $\mathrm{SNR} /$ symbol/branch associated with a larger signaling constellation.

In Figs. 5 and 6, the ASER performance curves for 16-ary PSK are plotted as a function of mean SNR/symbol per branch, for both the ideal $(\rho=1)$ and nonideal $(\rho=0.97)$ $M / 7-\mathrm{SC} / \mathrm{MRC}$ receivers. Observe that for small and moderate values of the mean SNR/symbol/branch, these ASER curves exhibit the familiar "waterfall slope." However, for large $\bar{\gamma}$, ASER knee behavior prevails for all the nonideal SC/MRC receiver structures. Notice that only in the case of perfect combining $(\rho=1)$, will the decay rate of the ASER for a large average $\mathrm{SNR} /$ symbol/branch will be proportional to $1 / \bar{\gamma}^{L}$, regardless of the value of $M$. This observation can be readily explained by performing an asymptotic analysis on (27)

$$
\begin{aligned}
\lim _{\bar{\gamma} \rightarrow \infty} \phi_{\gamma}(s) & =\lim _{\bar{\gamma} \rightarrow \infty} \frac{1}{(s \bar{\gamma})^{L}(1+1 / s \bar{\gamma})^{M-1}} \prod_{l=M}^{L} \frac{1}{1 / s \bar{\gamma}+M / l} \\
& =\frac{1}{(s \bar{\gamma})^{L}} \prod_{l=M}^{L}(l / M) .
\end{aligned}
$$

Comparison between Figs. 5 and 6 suggests that the Gaussian weighting errors affect the reduced complexity receiver structure (i.e., $M<L)$ more adversely than the $\operatorname{MRC}(M=L)$ receiver. As such, one may have to combine an additional number of diversity branches (and therefore incur additional complexity and cost) in the absence of perfect channel gain estimates, so as to achieve an adequate performance. For instance, from Fig. 5, we find that by only combining the five strongest multipaths, the reduced complexity receiver yields comparable performance to that of the MRC rake receiver which combines all the seven resolvable multipaths. However, if the channel gain estimates are not perfect (e.g., $\rho=0.97$ ), one may have to combine an additional multipath in order to attain a comparable performance to the nonideal MRC rake receiver, specifically at the lower ASER region (e.g., $\bar{P}_{e}=10^{-9}$ ), due to the knee behavior in the ASER curves.

Fig. 7 investigates the effects of Gaussian weighting error on the normalized mean combined SNR/symbol as a function of the number of available resolvable multipaths $L$ in a rake receiver for different number of $M$ strongest combined paths and $\rho$. For a fixed $M$, the normalized mean combined SNR $\bar{\gamma}_{g s c} / \bar{\gamma}$ increases with increasing $L$. However, the relative increase declines gradually as the difference between $L$ and $M$ gets larger, which is typical of a selection diversity system. Similarly, for a fixed $L$, increasing $M$ leads to a higher $\bar{\gamma}_{g s c} / \bar{\gamma}$, as expected. The percentage of improvement is more pronounced when $M$ is increased from one to two compared with going from $M=7$ to $M=8$. Observe also that for a fixed $M$, the degradation in the normalized mean combined SNR/symbol (due to weighting error) becomes more appreciable as $L$ increases. Although not explicitly shown in the figures, we found that the weighting errors affect the average combined SNR negligibly in comparison to their effect on the ASER performance as well as the PDF and CDF of the combiner 

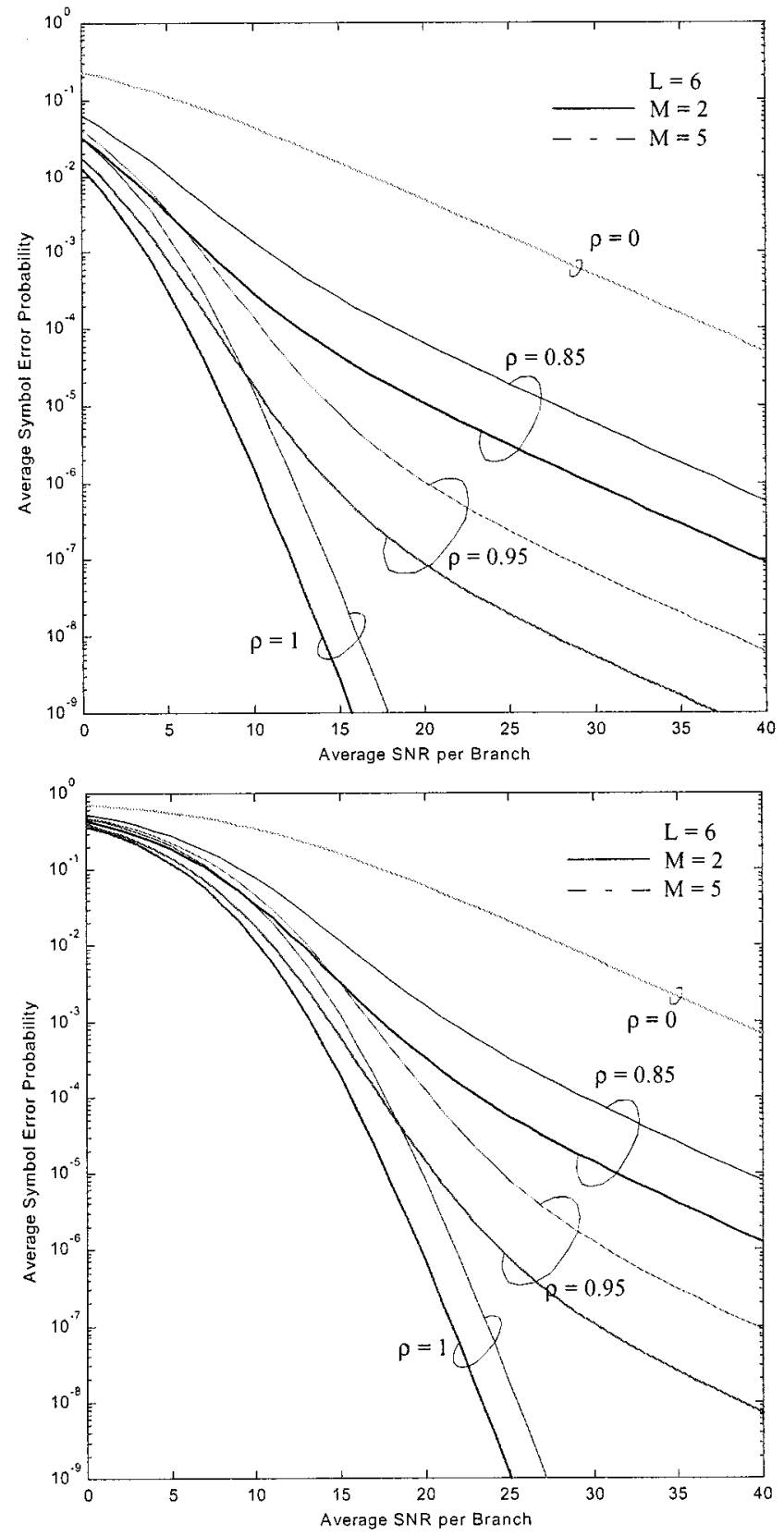

Fig. 4. Average symbol error rate performance of $\pi / 4-D Q P S K$ and 8-DPSK modulation schemes employing a nonideal SC/MRC receiver.

output SNR. For instance $\bar{\gamma}_{g s c}$, for 3/5-SC/MRC receiver has only dropped $10 \log _{10}(4.35 / 3.71)=0.7 \mathrm{~dB}$ when $\rho=0.9$ relative to that for $\rho=1$. But the same amount of weighting error can radically alter the PDF and CDF curves (see Figs. 2 and 3). Assuming $\rho=0.85$, it is straight-forward to show that $\bar{\gamma}_{g s c}$ for the nonideal 5/6-SC/MRC combiner has only dropped $10 \log _{10}(5.83 / 4.49)=1.1 \mathrm{~dB}$ relative to the perfect combining case. However, Fig. 4 shows that this severely degrades the ASER performance because the penalty in the average SNR/symbol/branch at $\bar{P}_{e}=10^{-5}$ is considerably larger than the drop in $\bar{\gamma}_{g s c}$.

Finally, Figs. 8 and 9 examine the effect of imperfect branch gain estimates in an energy-sharing multichannel binary DPSK system. It is important to note that in this case the total SNR

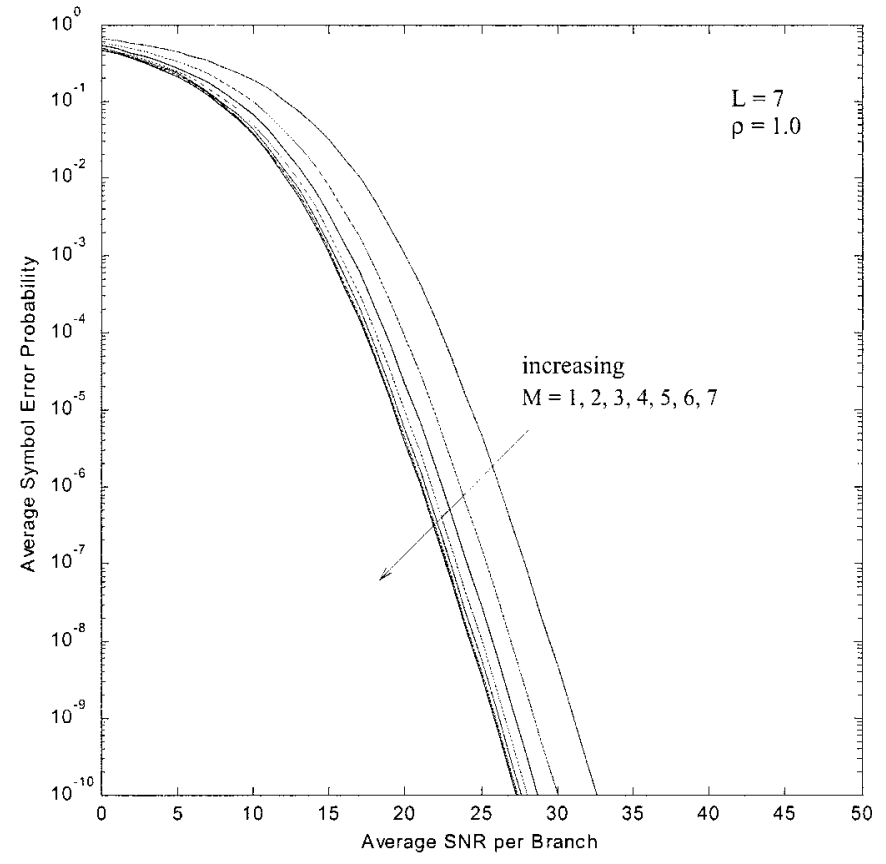

Fig. 5. Average symbol error rate performance of 16-ary coherent PSK for a "family" of diversity receiver structures over Rayleigh fading.

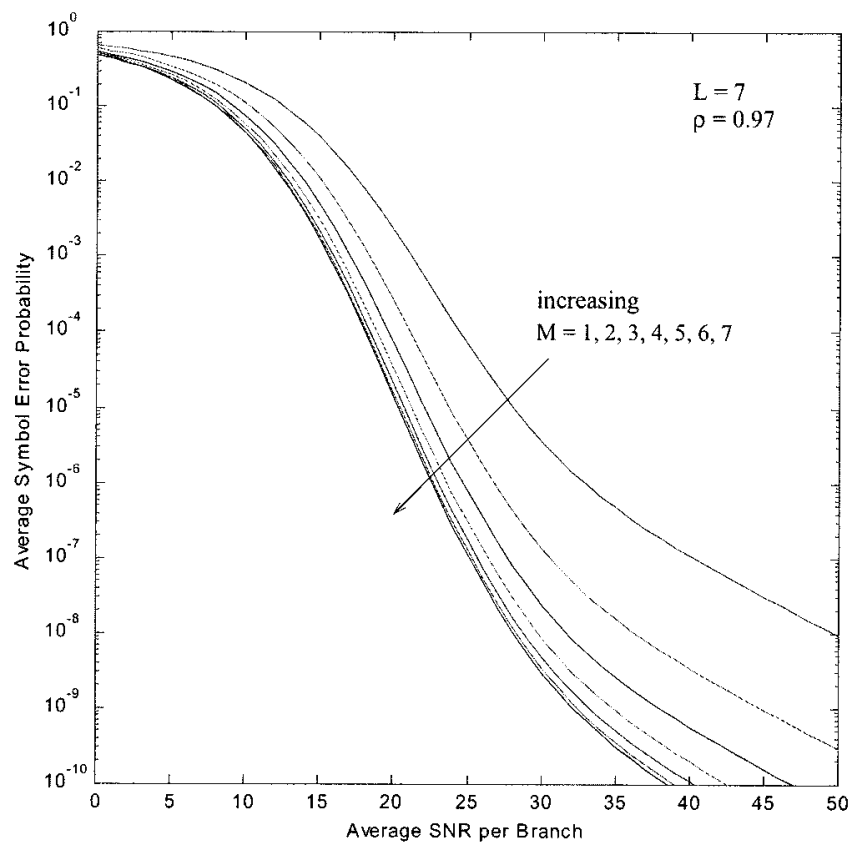

Fig. 6. Degradation of ASER performance of 16-ary coherent PSK due to Gaussian weighting error.

$\bar{\gamma}_{T}=L \bar{\gamma}$ is fixed and the average SNR/symbol/channel needs to be reduced as $L$ increases in order to maintain the same $\bar{\gamma}_{T}$. From Fig. 8, we observe that the weighting errors can cause the system to behave very differently depending on the value of $\rho$. For $\rho \ll 1$ (e.g., $\rho<0.3$ ), it is evident that the optimal number of diversity branches is $L=1$. This observation suggests that with very poor channel estimates, the combination losses will outweigh the benefits obtained by having additional diversity branches. At the other extreme (e.g., $\rho>0.9$ ), the diversity gain prevails and there is no optimal number of diversity 


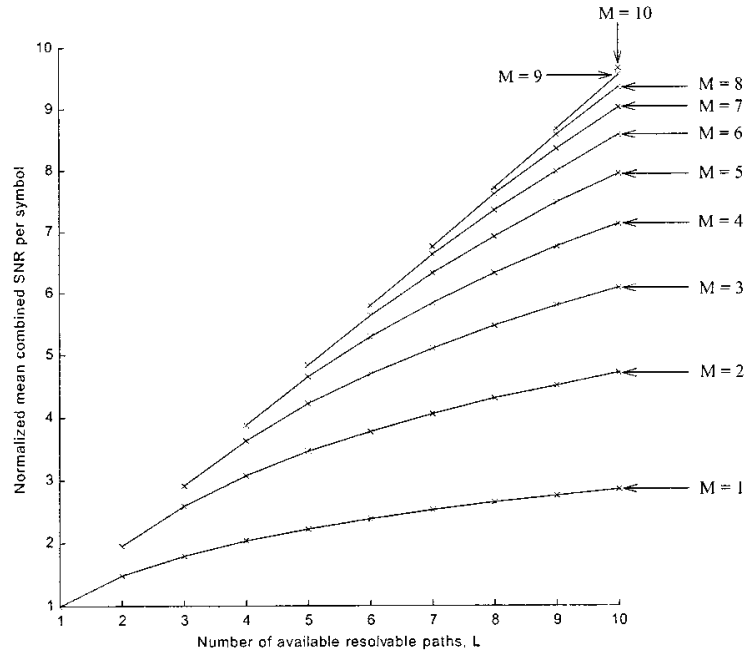

(a)

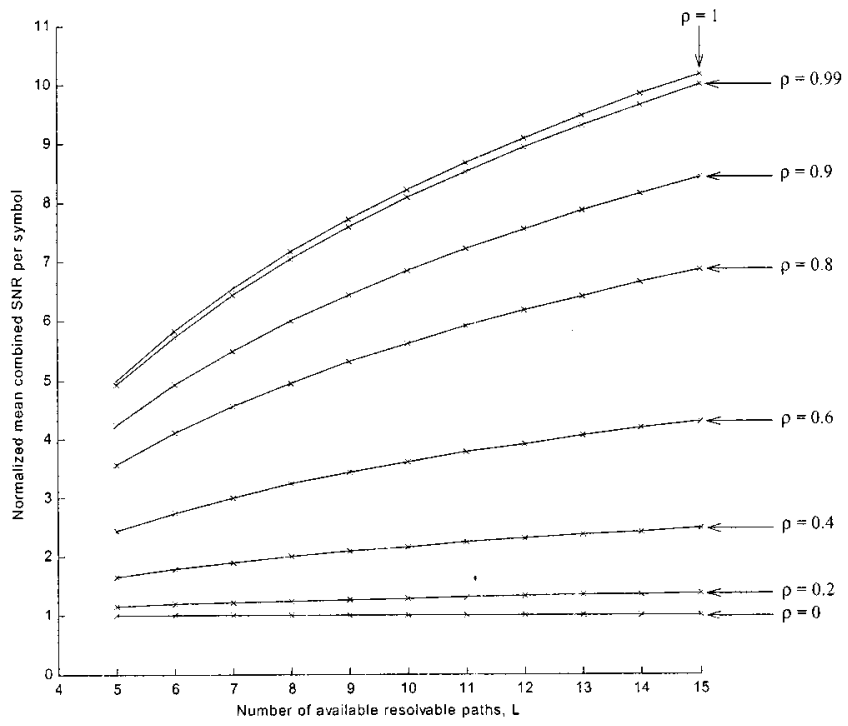

(b)

Fig. 7. The effects of weighting errors on the average combined SNR of an SC/MRC receiver. (a) $\rho=0.98$. (b) $M=5$.

branches that minimizes the ASER. For other values of $\rho$, we found that the ASER increases initially with increasing $L$ but declines rapidly afterwards (for large $L$ ). As such, systems designers should carefully choose an appropriate value for $L$ so that the multichannel communication performance will not be inferior to that for a single channel case. It should be pointed out, however, for a fixed $L$, increasing $M$ will always improve the ASER performance (see Fig. 9).

\section{CONCLUSION}

This paper first derives the statistics of an $M / L-\mathrm{SC} / \mathrm{MRC}$ combiner output SNR on Rayleigh fading channels in situations where the channel gain estimates are noisy. The application of these statistics (i.e., the PDF, MGF, and CDF of combiner output SNR) for characterizing the performance of diversity systems in terms of the ASER, outage rate of error probability and average combined SNR, is also presented. The MGF is used to

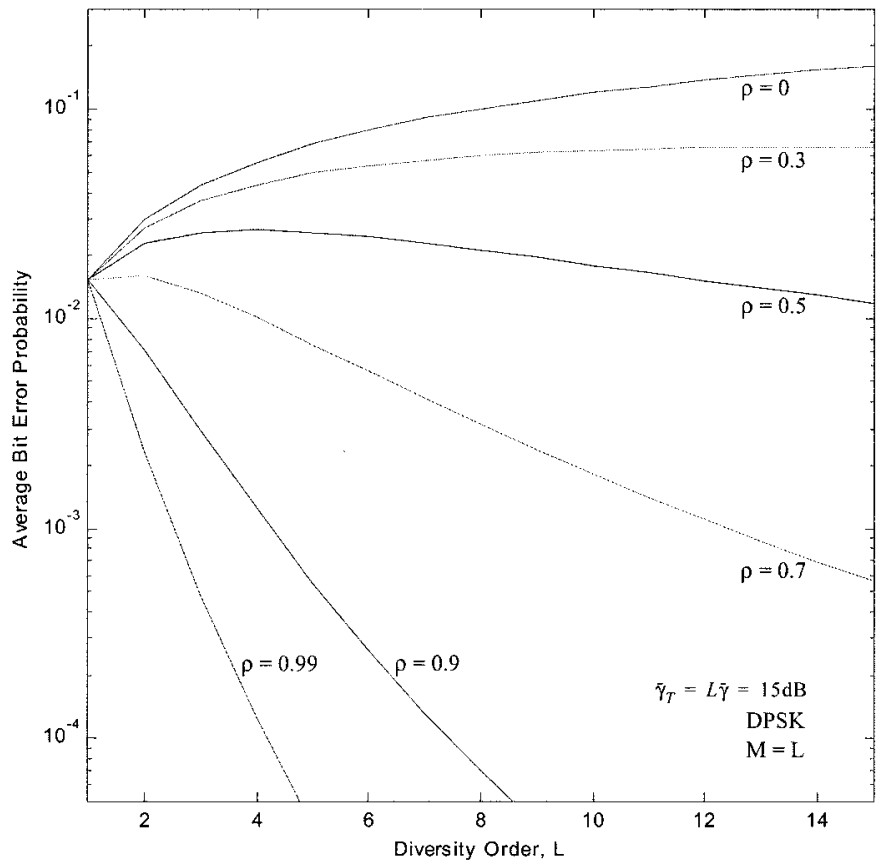

Fig. 8. Effect of imperfect branch gain estimates in an energy-sharing multichannel communication systems, given that $M=L$ (i.e., maximal-ratio diversity receiver).

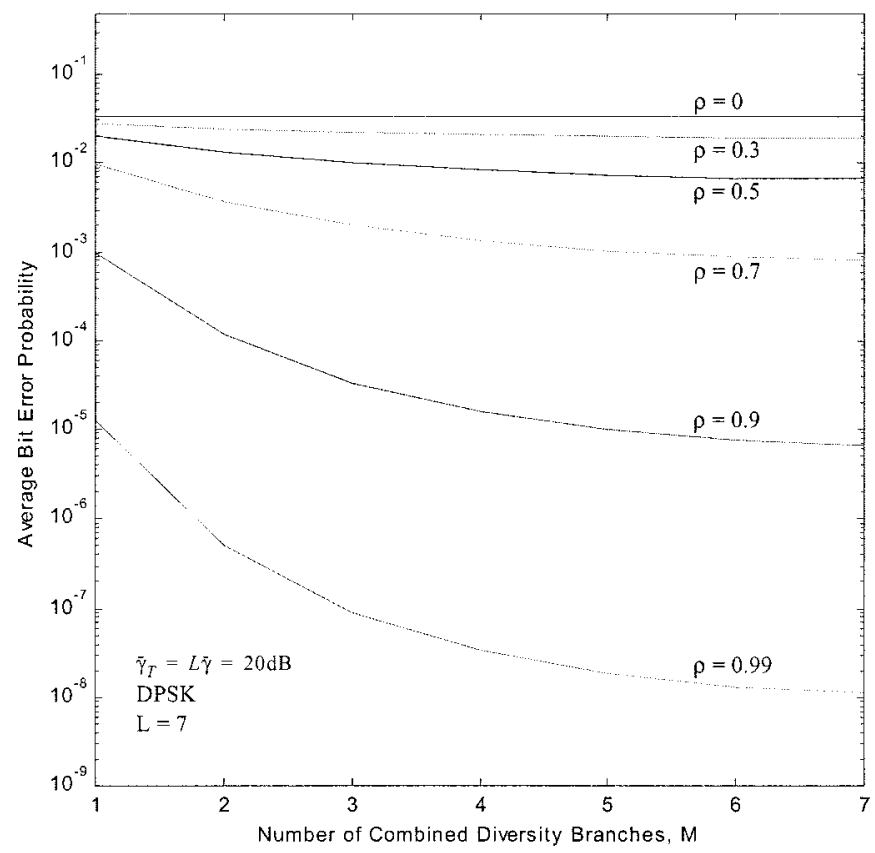

Fig. 9. Effect of imperfect branch gain estimates in an energy-sharing multichannel binary communications for a fixed diversity order $L$.

derive closed-form ASER expressions for a multitude of digital modulation schemes that are taken in conjunction with a "family" of receiver structures (with SC and MRC as limiting cases). The outage probability (in closed-form) can be computed readily using the CDF. A simple, closed-form expression for the average combined SNR can be derived using either the PDF or MGF. Selected numerical examples are provided in order to demonstrate the existence of an optimum number of diversity branches for an energy-sharing communications system. This 
optimum achieves a balance between the diversity gain and the combination losses (arising from imperfect combining) with additional number of diversity branches given that the received total SNR is fixed. Finally, we will conclude by noting that our general expressions for the PDF, CDF, and MGF of the SNR can be used in the analysis of several other applications of interest. Some examples include investigation into the efficacy of a hybrid SC/MRC antenna array in millimeter-wave, broadband indoor wireless communications; investigation into the performance of reduced-complexity, rake receiver structures in wideband code-division multiple-access systems; and analysis of a simple packet combining scheme to increase the throughput of packet radio networks. No doubt many other possible applications exist for these general expressions.

\section{APPENDIX I}

In this appendix, we will sketch of the derivations used for the PDF of random variable $Z$ shown in (6). The MGF of a sum of ordered, exponential random variables can be obtained using either the technique suggested by Sukhatme [16] (details can also be found in [9] and [17]) or a virtual-branch approach, which is outlined in [8]. Since the PDF of signal power in a Rayleigh fading channel is an exponential random variable, it is possible to demonstrate that the MGF of $Z$ is

$$
\begin{aligned}
\phi_{Z}(s) & =\frac{1}{\left(1+2 s \sigma^{2}\right)^{M-1}} \prod_{l=0}^{L-M} \frac{1}{1+\frac{2 s \sigma^{2} M}{l+M}} \\
& =\prod_{l=0}^{L-M} \frac{1}{\left[1+\frac{2 s \sigma^{2} M}{l+M}\right]^{D_{l}}} \\
& =\sum_{l=0}^{L-M} \sum_{k=1}^{D_{l}} \frac{\mu_{l}^{\left(D_{l}-k\right)}}{\left[1+\frac{2 s \sigma^{2} M}{l+M}\right]^{k}}
\end{aligned}
$$

where parameters $D_{l}$ and $\rho^{2}$ are defined as in (6), and the coefficient of the partial fraction expansion is given by

$$
\begin{aligned}
\mu_{l}^{\left(D_{l}-k\right)} & =\frac{1}{\left(D_{l}-k\right) !}\left(\frac{l+M}{2 \sigma^{2} M}\right)^{D_{l}-k} \frac{d^{\left(D_{l}-k\right)}}{d t} \\
\times & {\left.\left[\prod_{i=0, i \neq l}^{L-M} \frac{1}{\left(1+\frac{2 \sigma^{2} t M}{i+M}\right)^{D_{i}}}\right]\right|_{t=(-(l+M)) / 2 \sigma^{2} M} . }
\end{aligned}
$$

Note that the first product form on the right-side of (46) becomes identical to [9, eq. (12)] when the index is changed to $l^{\prime}=l+M$, and $2 \sigma^{2} \rightarrow \bar{\gamma}$ and $M \rightarrow L_{c}$ are replaced. It is also possible to rewrite (46) as

$$
\phi_{Z}(s)=\sum_{k=1}^{M} \frac{\mu_{0}^{(M-k)}}{\left(1+2 s \sigma^{2}\right)^{k}}+\sum_{l=1}^{L-M} \frac{\mu_{l}^{(0)}}{1+\frac{2 s \sigma^{2} M}{l+M}} .
$$

Therefore, it is evident that only the coefficients $\mu_{0}^{(M-k)}$ and $\mu_{l}^{(0)}$ need to be computed in (46). Fortunately, as we will show next, these can be evaluated in closed-form, thereby eliminating the need to numerically perform $\left(D_{l}-k\right)$ th order derivative of a product term with respect to $t$. Letting $D_{l}-k=0$ in (47), we have

$$
\mu_{l}^{(0)}=\prod_{i=0, i \neq l}^{L-M}\left(\frac{i+M}{i-l}\right)^{D_{i}} .
$$

When $l=0$, (49) reduces

$$
\mu_{0}^{(0)}=\prod_{i=1}^{L-M} \frac{i+M}{i}=\left(\begin{array}{c}
L \\
M
\end{array}\right)
$$

where $\left(\begin{array}{l}a \\ b\end{array}\right)=a ! /(b !(a-b) !)$ denotes the binomial coefficient. Similarly, for a positive integer $l \geq 1$, we get

$$
\begin{aligned}
\mu_{l}^{(0)} & =\left(\frac{M}{-l}\right)^{M} \prod_{i=0, i \neq l}^{L-M} \frac{i+M}{i-l} \\
& =\left(\frac{M}{l}\right)^{M} \frac{(-1)^{M+l-1}}{l+M} \frac{L !}{M !(l-1) !(L-M-l) !} \\
& =(-1)^{M+l-1}\left(\frac{l}{l+M}\right)\left(\frac{M}{l}\right)^{M}\left(\begin{array}{c}
L \\
M
\end{array}\right)\left(\begin{array}{c}
L-M \\
l
\end{array}\right)
\end{aligned}
$$

which is equivalent to (8).

To compute $\mu_{0}^{(M-k)}$, we must first rewrite (47) as

$$
\begin{aligned}
\mu_{0}^{(M-k)}= & \frac{1}{(M-k) !}\left(\frac{l+M}{2 \sigma^{2} M}\right)^{M-k} \frac{d^{(M-k)}}{d t} \\
& \times\left.\left[\sum_{i=1}^{L-M} \frac{C_{i}}{1+\frac{2 \sigma^{2}+M}{i+M}}\right]\right|_{t=(-(l+M)) / 2 \sigma^{2} M} \\
& \text { if } k<M<L
\end{aligned}
$$

where the coefficient of the partial fraction expansion $C_{i}$ is given by

$$
\begin{aligned}
C_{i} & =\left.\prod_{w=1, w \neq i}^{L-M} \frac{1}{\left(1+\frac{2 \sigma^{2} t M}{w+M}\right)}\right|_{t=(-(i+M)) / 2 \sigma^{2} M} \\
& =\prod_{w=1, w \neq i}^{L-M}\left(\frac{w+M}{w-i}\right) \\
& =(-1)^{i-1}\left(\frac{i}{i+M}\right)\left(\begin{array}{c}
L \\
M
\end{array}\right)\left(\begin{array}{c}
L-M \\
i
\end{array}\right) .
\end{aligned}
$$

Now, performing the differentiation in (52) and some algebraic manipulations, we obtain

$$
\mu_{0}^{(M-k)}=\sum_{i=1}^{L-M} C_{i}\left(\frac{i+M}{i}\right)\left(\frac{M}{-i}\right)^{M-k}, \quad k<M<L
$$

which simplifies to (9). When $M=L$, we find $\mu_{0}^{(L-k)}=0$ if $k<L$, and $\mu_{0}^{(L-k)}=1$ if $k=L$. Finally, the PDF of $Z$ shown in (6) can be immediately obtained by taking the inverse Laplace transform of the last MGF expression in (46). 


\section{APPENDIX II}

In this appendix, we will briefly highlight the important, intermediate steps in the derivation of the PDF of the SNR, shown in (7), from (4). Substituting (5) and (6) into (4) and after making some rearrangement, we obtain

$$
\begin{aligned}
p_{\gamma}(\gamma)= & \sum_{l=0}^{L-M} \sum_{k=1}^{D_{l}} \mu_{l}^{\left(D_{l}-k\right)} \frac{N}{(k-1) ! \lambda_{l}^{k} \sigma_{0}^{2}} \exp \left(\frac{-N \gamma}{\sigma_{0}^{2}}\right) \\
& \times \int_{0}^{\infty} Z^{k-1} \exp \left[-Z\left(\frac{\lambda_{l}+2 \sigma_{0}^{2}}{2 \sigma_{0}^{2} \lambda_{l}}\right)\right] \\
& \times I_{0}\left(\frac{\sqrt{2 N \gamma Z}}{\sigma_{0}^{2}}\right) d Z .
\end{aligned}
$$

The integral right side can be evaluated in closed-form using (56) (obtained using [19, eqs. (6.643.2) and (9.220.2)])

$$
\begin{aligned}
\int_{0}^{\infty} & x^{\mu-(1 / 2)} \exp (-\alpha x) I_{2 \nu}(2 \beta \sqrt{x}) d x \\
= & \frac{\Gamma\left(\mu+\nu+\frac{1}{2}\right)}{\Gamma(2 \nu+1) \beta \alpha^{\mu}}\left(\frac{\beta^{2}}{\alpha}\right)^{\nu+(1 / 2)} \\
& \times{ }_{1} F_{1}\left(\nu+\mu+\frac{1}{2}, 2 \nu+1 ; \frac{\beta^{2}}{\alpha}\right)
\end{aligned}
$$

where ${ }_{1} F_{1}(\cdot, \cdot ; \cdot)$ represents the confluent hypergeometric series. Subsequently, (55) simplifies into

$$
\begin{array}{r}
p_{\gamma}(\gamma)=\sum_{l=0}^{L-M} \sum_{k=1}^{D_{l}} \mu_{l}^{\left(D_{l}-k\right)} \frac{N}{\sigma_{0}^{2}} \exp \left(\frac{-N \gamma}{\sigma_{0}^{2}}\right)\left(\frac{2 \sigma_{0}^{2}}{\lambda_{l}+2 \sigma_{0}^{2}}\right)^{k} \\
\times_{1} F_{1}\left(k, 1 ; \frac{N \gamma \lambda_{l}}{\left(\lambda_{l}+2 \sigma_{0}^{2}\right) \sigma_{0}^{2}}\right)
\end{array}
$$

Recognizing that the ${ }_{1} F_{1}(\cdot, \cdot ; \cdot)$ function in $(57)$ can be replaced by a finite polynomial, Kummer's transformation formula [19, eq. (9.212.1)] is applied and, we obtain

$$
\begin{aligned}
p_{\gamma}(\gamma)= & \sum_{l=0}^{L-M} \sum_{k=1}^{D_{l}} \mu_{l}^{\left(D_{l}-k\right)} \frac{N}{\sigma_{0}^{2}} \\
& \times \exp \left(\frac{-2 N \gamma}{\lambda_{l}+2 \sigma_{0}^{2}}\right)\left(\frac{2 \sigma_{0}^{2}}{\lambda_{l}+2 \sigma_{0}^{2}}\right)^{k} \\
& \times \sum_{n=0}^{k-1} \frac{1}{n !}\left(\begin{array}{c}
k-1 \\
n
\end{array}\right)\left[\frac{N \gamma \lambda_{l}}{\left(\lambda_{l}+2 \sigma_{0}^{2}\right) \sigma_{0}^{2}}\right]^{n} .
\end{aligned}
$$

Next, we need to make appropriate substitutions for $\rho_{0}^{2}$ and $\rho^{2}$ in (58) by relating these parameters to the average SNR per branch $\bar{\gamma}$ and the magnitude of the normalized correlation coefficient $\rho$. From the definition of the average SNR per branch, we have

$$
\bar{\gamma}=\frac{\frac{1}{2}\left\langle g_{k} g_{k}^{*}\right\rangle}{N}=\frac{\sigma_{x}^{2}}{N}
$$

Since $\rho$ is the magnitude of the complex correlation between $p_{k}$ and $g_{k}$, which are normalized to their autocorrelation values, we also have the following relation:

$$
\rho^{2}=\left|\frac{\left\langle p_{k} g_{k}^{*}\right\rangle}{\left\langle p_{k} p_{k}^{*}\right\rangle}\right|^{2}=\frac{R_{c}^{2}+R_{c s}^{2}}{\sigma_{x}^{4}}=\frac{\sigma^{2}}{\sigma_{x}^{2}} \Leftrightarrow \sigma^{2}=\rho^{2} \sigma_{x}^{2}
$$

Using (60), the variance $\rho_{0}^{2}$ can be restated as

$$
\begin{aligned}
\sigma_{0}^{2} & =\frac{\sigma_{x}^{4}-\left(R_{c}^{2}+R_{c s}^{2}\right)}{\sigma_{x}^{2}}=\sigma_{x}^{2}\left(1-\frac{R_{c}^{2}+R_{c s}^{2}}{\sigma_{x}^{4}}\right) \\
& =\sigma_{x}^{2}\left(1-\rho^{2}\right) .
\end{aligned}
$$

Finally, substituting (59)-(61) into (58) leads to the desired result

$$
\begin{aligned}
p_{\gamma}(\gamma)= & \sum_{l=0}^{L-M} \sum_{k=1}^{D_{l}} \mu_{l}^{\left(D_{l}-k\right)} \frac{1}{\bar{\gamma}\left(1-\rho^{2}\right)} \\
& \times \exp \left[\frac{-(l+M) \gamma}{\bar{\gamma}\left(M+l\left(1-\rho^{2}\right)\right)}\right]\left[\frac{(l+M)\left(1-\rho^{2}\right)}{M+l\left(1-\rho^{2}\right)}\right]^{k} \\
& \times \sum_{n=0}^{k-1} \frac{1}{n !}\left(\begin{array}{c}
k-1 \\
n
\end{array}\right)\left[\frac{\gamma \rho^{2} M}{\bar{\gamma}\left(1-\rho^{2}\right)\left[M+l\left(1-\rho^{2}\right)\right]}\right]^{n}
\end{aligned}
$$

which is equivalent to (7).

\section{REFERENCES}

[1] N. Kong, T. Eng, and L. B. Milstein, "Selection combining scheme for rake receivers," in Proc. IEEE ICUPC'95, Oct. 1995, pp. 426-430.

[2] T. Eng, N. Kong, and L. B. Milstein, "Comparison of diversity combining techniques for Rayleigh fading channels," IEEE Trans. Commun., vol. 44, pp. 1117-1129, Sept. 1996.

[3] K. J. Kim, S. Y. Kwon, E. K. Hong, and K. C. Whang, "Comments on comparison of diversity combining techniques for Rayleigh fading channel," IEEE Trans. Commun., vol. 46, pp. 1109-1110, Sept. 1998.

[4] R. Wong, A. Annamalai, and V. K. Bhargava, "Evaluation of predetection diversity techniques for rake receivers," in Proc. IEEE PACRIM'97, Aug. 1997, pp. 227-230.

[5] M.-S. Alouini and M. K. Simon, "Performance of coherent receivers with hybrid SC/MRC over Nakagami-m fading channels," IEEE Trans. Veh. Technol., vol. 48, pp. 1155-1164, July 1999.

[6] _ , "Application of the Dirichlet transformation to the performance evaluation of generalized selection combining over Nakagami-m fading channels," J. Commun. Networks, vol. 1, pp. 5-13, Mar. 1999.

[7] A. Annamalai, C. Tellambura, and V. K. Bhargava, "Analysis of hybrid diversity systems on fading channels," in Proc. IEEE ISWC'99, June 1999, pp. 70-71.

[8] M. Win and J. Winters, "Analysis of selection/maximal-ratio combining in Rayleigh fading," in Proc. IEEE ICC'99, June 1999, pp. 6-10.

[9] M.-S. Alouini and M. K. Simon, "An MGF-based performance analysis of generalized selection combining over Rayleigh fading channels," IEEE Trans. Commun., vol. 48, pp. 401-414, Mar. 2000.

[10] P. Bello and B. D. Nelin, "Predetection diversity combining with selectively fading channels," IRE Trans. Commun. Syst., vol. 10, pp. 32-42, Jan. 1962

[11] M. Schwartz, W. R. Bennett, and S. Stein, Communication Systems and Techniques. New York: McGraw-Hill, 1966.

[12] J. G. Proakis, "Probabilities of error for adaptive reception of M-phase signals," IEEE Trans. Commun. Technol., vol. 16, pp. 71-81, Jan. 1968.

[13] M. J. Gans, "The effect of Gaussian error in maximal ratio combiners," IEEE Trans. Commun. Technol., vol. 19, pp. 492-500, Aug. 1971.

[14] B. Tomiuk, N. C. Beaulieu, and A. Abu-Dayya, "General forms for maximal ratio diversity with weighting errors," IEEE Trans. Commun., vol. 47, pp. 488-492, Apr. 1999.

[15] A. Annamalai and C. Tellambura, "Error rates for Nakagami-m fading multichannel reception of binary and $M$-ary signals," IEEE Trans. Commun., vol. 49, pp. 58-68, Jan. 2001.

[16] P. V. Sukhatme, "Tests of significance for samples of the $\chi^{2}$ population with two degrees of freedom," Annals Eugenics, vol. 8, pp. 52-56, 1937.

[17] B. C. Arnold, N. Balakrishnan, and H. N. Nagaraja, A First Course in Order Statistics. New York: Wiley, 1992.

[18] A. Papoulis, Probability, Random Variables and Stochastic Processes. New York: McGraw-Hill, 1991.

[19] I. S. Gradshteyn and I. M. Ryzhik, Table of Integrals, Series and Products. New York: Academic, 1994 
[20] C. Tellambura, A. J. Mueller, and V. K. Bhargava, "Analysis of $M$-ary phase-shift-keying with diversity reception for land-mobile satellite channels," IEEE Trans. Veh. Technol., vol. 46, pp. 910-922, Nov. 1997.

[21] J. W. Craig, "A new, simple and exact result for calculating the probability of error for two dimensional signal constellations," in Proc. IEEE Milcom'91 Conf. Record, 1991, pp. 25.5.1-25.5.5.

[22] X. Dong, N. C. Beaulieu, and P. H. Wittke, "Error probability of twodimensional $M$-ary signaling in fading," IEEE Trans. Commun., vol. 47, pp. 352-355, Mar. 1999.

[23] C. Tellambura and A. Annamalai, "Tutorial notes: Analytical tools for wireless communications system design," in Proc. IEEE VTC'00 (Spring), Tokyo, May 2000.

[24] J. Ritchey and M. Azizoglu, "Performance analysis of generalized selection combining with switching constraints," IEEE Commun. Lett., vol. 4, pp. 152-154, May 2000

[25] A. Annamalai and C. Tellambura, "The effects of weighting errors in hybrid SC/MRC receivers," in Proc. IEEE Wireless Communications and Networking Conf., Sept. 2000, pp. 211-215.

[26] W. B. Davenport, Jr. and W. L. Root, An Introduction to the Theory of Random Signals and Noise. New York: IEEE Press, 1987, pp. 158-167.

A. Annamalai received the B.Eng. degree with the highest distinction from the University of Science of Malaysia, Malaysia, in 1993, and the M.A.Sc. and Ph.D. degrees from the University of Victoria, Canada, in 1997 and 1999, respectively, all in electrical and computer engineering.

Currently, he is with the Bradley Department of Electrical and Computer Engineering of Virginia Polytechnic Institute and State University, Alexandria, VA, as an Assistant Professor and an Associate Director of the Mobile and Portable Radio Research Group. He was a radio frequency design engineer with Motorola, Malaysia, from 1993 to 1995. His current research interests are in high-speed data transmission over wireless links, adaptive modulation and coding, smart antenna, OFDM, and wireless communication theory.

Dr. Annamalai was awarded the 2001 IEEE Leon K. Kirchmayer Prize Paper Award for his work on diversity systems. He is an Editor for the IEEE TRANSACTIONS ON WIRELESS COMMUNICATIONS, an Associate Editor for the IEEE COMmunicATIONS LetTers and is the Technical Program Chair of the IEEE VTC2002 (Fall).
C. Tellambura (M'97) received the B.Sc. degree with honors from the University of Moratuwa, Sri Lanka, in 1986, the M.Sc. degree in electronics from the King's College, U.K., in 1988, and the Ph.D. degree in electrical engineering from the University of Victoria, Canada, in 1993.

He was a Postdoctoral Research Fellow with the University of Victoria and the University of Bradford. Currently, he is a Senior Lecturer at Monash University, Australia. His research interests include coding, communications theory, modulation, equalization, and wireless communications.

Dr. Tellambura is an Editor for the IEEE TRANSACTIONS ON Communications and the IEEE Transactions ON Wireless COMMUNICATIONS 